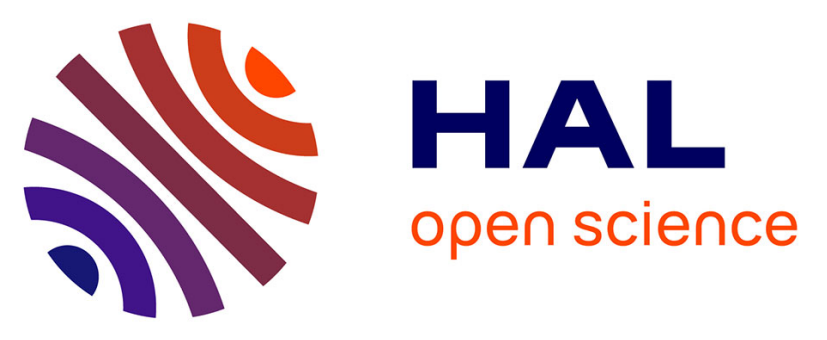

\title{
Atxn2-CAG100-KnockIn mouse spinal cord shows progressive TDP43 pathology associated with cholesterol biosynthesis suppression
}

Júlia Canet-Pons, Nesli-Ece Sen, Aleksandar Arsović, Luis-Enrique

Almaguer-Mederos, Melanie Halbach, Jana Key, Claudia Döring, Anja

Kerksiek, Gina Picchiarelli, Raphaelle Cassel, et al.

\section{To cite this version:}

Júlia Canet-Pons, Nesli-Ece Sen, Aleksandar Arsović, Luis-Enrique Almaguer-Mederos, Melanie Halbach, et al.. Atxn2-CAG100-KnockIn mouse spinal cord shows progressive TDP43 pathology associated with cholesterol biosynthesis suppression. Neurobiology of Disease, 2021, 152, pp.105289. 10.1016/j.nbd.2021.105289 . inserm-03376340

\section{HAL Id: inserm-03376340 https://www.hal.inserm.fr/inserm-03376340}

Submitted on 13 Oct 2021

HAL is a multi-disciplinary open access archive for the deposit and dissemination of scientific research documents, whether they are published or not. The documents may come from teaching and research institutions in France or abroad, or from public or private research centers.
L'archive ouverte pluridisciplinaire HAL, est destinée au dépôt et à la diffusion de documents scientifiques de niveau recherche, publiés ou non, émanant des établissements d'enseignement et de recherche français ou étrangers, des laboratoires publics ou privés. 


\title{
Atxn2-CAG100-KnockIn mouse spinal cord shows progressive TDP43 pathology associated with cholesterol biosynthesis suppression
}

\author{
Júlia Canet-Pons $^{\mathrm{a}, 1}$, Nesli-Ece Sen ${ }^{\mathrm{a}, \mathrm{b}, 1}$, Aleksandar Arsovićc ${ }^{\mathrm{a}}$, \\ Luis-Enrique Almaguer-Mederos $^{\mathrm{a}, \mathrm{c}}$, Melanie V. Halbach ${ }^{\mathrm{a}}$, Jana Key ${ }^{\mathrm{a}, \mathrm{b}}$, Claudia Döring ${ }^{\mathrm{d}}$, \\ Anja Kerksiek ${ }^{\mathrm{e}}$, Gina Picchiarelli ${ }^{\mathrm{f}}$, Raphaelle Cassel ${ }^{\mathrm{f}}$, Frédérique René ${ }^{\mathrm{f}}$, Stéphane Dieterlé ${ }^{\mathrm{f}}$, \\ Nina V. Fuchs ${ }^{g}$, Renate König ${ }^{g}$, Luc Dupuis ${ }^{\mathrm{f}}$, Dieter Lütjohann ${ }^{\mathrm{e}}$, Suzana Gispert ${ }^{\mathrm{a}}$, \\ Georg Auburger ${ }^{\text {a, }}$
}

a Experimental Neurology, Medical Faculty, Goethe University, 60590 Frankfurt am Main, Germany

${ }^{\mathrm{b}}$ Faculty of Biosciences, Goethe University, 60438 Frankfurt am Main, Germany

${ }^{\mathrm{c}}$ Center for Investigation and Rehabilitation of Hereditary Ataxias (CIRAH), Holguín, Cuba

d Dr. Senckenberg Institute of Pathology, Medical Faculty, Goethe University, 60590 Frankfurt am Main, Germany

${ }^{\mathrm{e}}$ Institute of Clinical Chemistry and Clinical Pharmacology, University Hospital Bonn, 53127 Bonn, Nordrhein-Westfalen, Germany

${ }^{\mathrm{f}}$ UMRS-1118 INSERM, Faculty of Medicine, University of Strasbourg, 67000 Strasbourg, France

${ }^{\mathrm{g}}$ Host-Pathogen Interactions, Paul-Ehrlich-Institute, 63225 Langen, Germany

\section{A R T I C L E I N F O}

\section{Keywords:}

Olivo-ponto-cerebellar atrophy

Tauopathy

Neuroinflammation

Demyelination

Leukoencephalopathy

Steroidogenesis

\begin{abstract}
A B S T R A C T
Large polyglutamine expansions in Ataxin-2 (ATXN2) cause multi-system nervous atrophy in Spinocerebellar Ataxia type 2 (SCA2). Intermediate size expansions carry a risk for selective motor neuron degeneration, known as Amyotrophic Lateral Sclerosis (ALS). Conversely, the depletion of ATXN2 prevents disease progression in ALS. Although ATXN2 interacts directly with RNA, and in ALS pathogenesis there is a crucial role of RNA toxicity, the affected functional pathways remain ill defined. Here, we examined an authentic SCA2 mouse model with Atxn2CAG100-KnockIn for a first definition of molecular mechanisms in spinal cord pathology. Neurophysiology of lower limbs detected sensory neuropathy rather than motor denervation. Triple immunofluorescence demonstrated cytosolic ATXN2 aggregates sequestrating TDP43 and TIA1 from the nucleus. In immunoblots, this was accompanied by elevated CASP3, RIPK1 and PQBP1 abundance. RT-qPCR showed increase of Grn, Tlr7 and Rnaset2 mRNA versus Eif5a2, Dcp2, Uhmk1 and Kif5a decrease. These SCA2 findings overlap well with known ALS features. Similar to other ataxias and dystonias, decreased mRNA levels for Unc80, Tacr1, Gnal, Ano3, Kcna2, Elovl5 and Cdr1 contrasted with Gpnmb increase. Preterminal stage tissue showed strongly activated microglia containing ATXN2 aggregates, with parallel astrogliosis. Global transcriptome profiles from stages of incipient motor deficit versus preterminal age identified molecules with progressive downregulation, where a cluster of cholesterol biosynthesis enzymes including Dhcr24, Msmo1, Idi1 and Hmgcs1 was prominent. Gas chromatography demonstrated a massive loss of crucial cholesterol precursor metabolites. Overall, the ATXN2 protein aggregation process affects diverse subcellular compartments, in particular stress granules, endoplasmic reticulum and receptor tyrosine kinase signaling. These findings identify new targets and potential biomarkers for neuroprotective therapies.
\end{abstract}

\section{Introduction}

Within the dynamic research field into neurodegenerative disorders, the rare monogenic variant SCA2 (Spinocerebellar Ataxia type 2) gained much attention over the past decade, since its pathogenesis is intertwined with the motor neuron diseases ALS/FTLD (Amyotrophic Lateral Sclerosis / Fronto-Temporal Lobar Dementia) and with extrapyramidal syndromes such as Parkinson/PSP (Progressive Supranuclear Palsy).

\footnotetext{
* Corresponding author.

E-mail address: auburger@em.uni-frankfurt.de (G. Auburger).

1 Joint first authorship.
} 
Particularly in the past years, it received massive interest since antisense-oligonucleotides were shown to effectively prevent the disease in mouse models, with clinical trials now being imminent (Becker et al., 2017; Scoles et al., 2017; Scoles and Pulst, 2019). Still, there is an urgent unmet need to define the molecular events of pathogenesis and to identify biomarkers of progression in SCA2 patients, which reflect therapeutic benefits much more rapidly than clinical rating scales, brain imaging volumetry or neurophysiology measures.

SCA2 was first described as separate entity in Indian patients, based on the characteristic early slowing of eye saccadic movements (Wadia and Swami, 1971). A molecularly homogeneous founder population of $\sim 1,000$ patients in Cuba made detailed clinical, neurophysiological and pathological analyses possible (Auburger et al., 1990; Auburger, 2012; Estrada et al., 1999; Orozco Diaz et al., 1990; Rodriguez-Labrada et al., 2019; Rub et al., 2013; Velazquez-Perez et al., 2004). The autosomal dominant inheritance was shown to be caused by unstable polyglutamine (polyQ) expansions in the Ataxin-2 gene (ATXN2) (Pulst et al., 1996), a stress-response factor conserved from yeast to plants and mammals (Auburger et al., 2017). The expansion size correlates with younger onset age and faster progression of disease, which manifests with weight loss (later enhanced by dysphagia), muscle cramps and deficient motor coordination (Almaguer-Mederos et al., 2010; Belal et al., 1994; Riess et al., 1997; Rub et al., 2006; Schols et al., 1997; Velazquez-Perez et al., 2014). Organisms lacking ATXN2 do not show immediate adverse effects, and are protected from several neurodegeneration variants with tauopathy, such as ALS/FTLD (Al-Ramahi et al., 2007; Becker et al., 2017; Elden et al., 2010; Ross et al., 2011; Shulman and Feany, 2003). However, in view of the association of ATXN2-deficiency with progressive obesity, hyperlipidemia and diabetes mellitus (Auburger et al., 2014; Lastres-Becker et al., 2008), it must be asked if such insidiously appearing metabolic excess signs would accompany any neuroprotective treatment by ATXN2 depletion.

PolyQ expansions in different proteins always cause progressive neurodegeneration, via the formation of protein aggregates. However, each polyQ disease shows a different pattern of pathology in the brain (Paulson et al., 2017; Scherzed et al., 2012), so features of each disease protein around the polyQ expansion determine which neuron populations are preferentially vulnerable. ATXN2 is strongly expressed in large neurons and normally localized at the rough endoplasmic reticulum (Fittschen et al., 2015; van de Loo et al., 2009) or at plasma membrane sites of receptor tyrosine kinase endocytosis (Drost et al., 2013; Nonis et al., 2008; Ralser et al., 2005b), due to protein interactions with PABPC1 and SRC, respectively. Possibly via its influence on endocytosis, an inhibition of mTOR signals, fat stores and cell size was shown for ATXN2 orthologs in yeast, worms and mice (Bar et al., 2016; DeMille et al., 2015; Lastres-Becker et al., 2016). Its role for the ribosomal translation machinery is unclear, since its absence does not change the polysome profile markedly (Lastres-Becker et al., 2016). During periods of cell damage, ATXN2 relocalizes to cytosolic stress granules where RNA quality control is performed, via recruitment of several nuclear proteins such as TIA1 (Ralser et al., 2005a). ATXN2 promotes stress granule formation and decreases P-body size (Nonhoff et al., 2007). The physiological interactions of ATXN2 with proteins and RNAs depend on its C-terminal PAM2 motif, its central Lsm and LsmAD domain, and its alternatively spliced proline-rich domain (Damrath et al., 2012; Lastres-Becker et al., 2019; Satterfield and Pallanck, 2006; Yokoshi et al., 2014), whereas its pathological aggregation is determined by the N-terminal polyQ domain. Currently it is unclear if ATXN2 aggregation occurs within stress granules, to what degree its physiological interactomes are disturbed in SCA2, and which molecular and functional deficits underlie the progressive neurodegeneration, as putative targets of neuroprotective therapies.

In model organisms such as yeast, flies and mice it was shown that ATXN2 depletion by genetic knockout or mRNA-knockdown protects against the neurotoxicity of TDP43, which causes motor neuron degeneration in ALS and in FTLD. In addition, patient studies showed genetic ATXN2 variants to contribute to the risk of ALS/FTLD (Becker et al., 2017; Elden et al., 2010; Gispert et al., 2012; Lahut et al., 2012; Lee et al., 2011; Rubino et al., 2019). Although the molecular mechanisms of ALS/FTLD are not understood, the crucial role of RNA toxicity in its pathogenesis became plain upon the identification of various ALS/ FTLD disease genes, such as the RNA binding proteins TDP43 (encoded by the TARDBP gene), FUS, HNRNPA2B1, the RNA-particle transporting KIF5A, the RNA toxicity sensor RIPK1, and other stress response factors such as GRN and SOD1 (Taylor et al., 2016).

We recently generated a new SCA2 mouse model via Atxn2-CAG100KnockIn (KIN) (Sen et al., 2019b) and are using it here to obtain initial insights into spinal cord pathology at the molecular and cellular level. Homozygous animals show somatic mosaicism of the expansion size, with maximal lifespan of 14 months. Gender differences are observed for the phenotype of initial weight gain at 5 weeks or 10 weeks. This is followed by insidious weight reduction, later impairment of rotarod performance at 20 weeks, and reduced vertical movements and vertical time in open field tests from 30 weeks of age. Their progressive loss of brain weight is accompanied by diminished levels of the abundant metabolite $\mathrm{N}$-acetylaspartate (NAA) upon magnetic resonance imaging, as in SCA2 patients. NAA is an energy storage compound synthesized by neuronal mitochondria to be used by oligodendroglia for the myelination of axons. Since human SCA2 autopsy material is very scarce and usually of insufficient quality for RNA studies, these animals provided the first opportunity to elucidate affected pathways and subcellular compartments, without confounding overexpression artifacts. Given that ATXN2 is an RNA-binding protein and that RNA toxicity is central for ALS pathogenesis, an unbiased global transcriptome survey at two different ages was performed.

Overall, our study defines molecular changes, which underlie afferent sensory and efferent motor pathology in spinal cord neurons, or provide mechanistic insights into glial changes. At global transcriptome level we identify the most dramatic progression events. Crucially, we demonstrate a severe deficit in steroidogenesis, which will eventually affect all cell types in nervous tissue. A clear overlap with the known pathomechanisms of ALS, as well as other ataxias and dystonias was observed.

\section{Materials and methods}

\subsection{Animal breeding}

Generation, housing, genotyping and dissection of the Atxn2CAG100-Knock-in mouse (Sen et al., 2019b) and Atxn2-Knock-out mice (Lastres-Becker et al., 2016) maintained in C57BL/6J background was previously described. During breeding, offspring were routinely genotyped around postnatal day 10 and immediately after weaning sex-/agematched pairs of WT and KIN animals were placed in the same cage, aged together, and studied in comparison, employing both sexes during all experiments. The study was ethically assessed by the Regierungspräsidium Darmstadt, with approval number V54-19c20/15-FK/ 1083.

Nerve conduction studies, electromyography and quantitative reverse-transcriptase PCR of lower limbs:

Mice at the age of $9-10$ months $(n=6$ for mutants and $n=7$ for controls) were anaesthetized with $100 \mathrm{mg} / \mathrm{kg}$ of ketamine chlorhydrate (Imalgene 1000; Merial, France) and $12 \mathrm{mg} / \mathrm{kg}$ of xylazine (Rompun 2\%; Bayer HealthCare, Loos, France) intraperitoneally. During the tests, mouse body temperature was kept constant between 34 and $36{ }^{\circ} \mathrm{C}$ by means of a thermostat-controlled heating pad. Neurophysiological and electromyography (EMG) recordings were made with a standard electroneuromyograph apparatus (AlpineBiomed ApS, Denmark) in accordance with the guidelines of the American Association of Electrodiagnostic Medicine. The low-pass filter was set at $20 \mathrm{~Hz}$, and the high-pass set at $10 \mathrm{kHz}$. These settings were used for all measurements.

For the tail sensory nerve response, stimulating electrodes placed 
distally and recording electrodes placed proximally were inserted in the tail $3 \mathrm{~cm}$ apart. A ground electrode was placed between the stimulating and the recording electrodes. The nerve was stimulated with squarewave pulses of $0.1 \mathrm{~ms}$ duration with a repetition rate set at $3 \mathrm{~Hz}$. Supramaximal responses were gradually generated, and maximal responses were obtained with stimulus currents $<2 \mathrm{~mA}$. Data were acquired with a sensitivity between 10 and $20 \mu \mathrm{V} /$ division with a sweep speed of $1 \mathrm{~ms} /$ division to ensure accurate measurement of lowamplitude responses. The latency was measured as the time from the given electrical stimulus to the appearance of a sensory nerve response corresponding to the initial deflection from the baseline. Baseline to peak amplitudes $(\mu \mathrm{V})$ from the sensory nerve-evoked responses were measured and averaged, resulting in one averaged amplitude per animal, which was used for statistical analysis.

Compound muscle action potentials (CMAP) were recorded in gastrocnemius muscle as described previously (Oosthuyse et al., 2001). Briefly, CMAPs were elicited with square pulses of $0.2 \mathrm{~ms}$ duration with a repetition rate set at $1 \mathrm{~Hz}$, delivered with a monopolar needle electrode to the sciatic nerve at the sciatic notch level. CMAPs were measured by two monopolar needle electrodes inserted in the gastrocnemius muscle, and the system was grounded by subcutaneously inserted monopolar needle electrode in the back of the animal. Data were acquired with a sensitivity between 1 and $5 \mathrm{mV}$ /division with a sweep speed of $1 \mathrm{~ms} /$ division to ensure accurate measurement of low-amplitude responses. Supramaximal responses were gradually generated, and maximal responses were obtained with stimulus currents $<5 \mathrm{~mA}$. The latency was measured as the time from the given electrical stimulus to the appearance of a muscle response - the initial CMAP negative deflection from the baseline. Amplitudes $(\mathrm{mV})$ from the baseline to the maximal negative peak of the CMAPs were measured and averaged, resulting in one average CMAP amplitude per animal, which was used for statistical analysis.

For electromyography recording, a monopolar needle electrode (diameter, $0.3 \mathrm{~mm}$; 9013R0313, Alpine Biomed ApS, Denmark) was inserted into the tail of the mouse to ground the system. Recordings were made in the gastrocnemius muscle, using a concentric needle electrode (diameter, $0.3 \mathrm{~mm}$; 9013S0012, AlpineBiomed ApS, Denmark). Electrical activity was monitored at least $2 \mathrm{~min}$ in three different sites. Spontaneous activity was differentiated from voluntary activity by visual inspection.

The RT-qPCR analysis was done in Tibialis Anterior and Soleus muscles from 4 mice per genotype. Muscles were harvested, rapidly frozen in liquid nitrogen and stored at $-80^{\circ} \mathrm{C}$ until use. Frozen tissues were placed into tubes containing a $5 \mathrm{~mm}$ stainless steel bead (Qiagen, Courtaboeuf, France) and $1 \mathrm{ml}$ of Trizol reagent (Life Technologies), and homogenized using a TissueLyser (Qiagen). RNA was prepared from tissue homogenenates following Trizol manufacturer's instructions. One microgram of total RNA was used to synthesize cDNA using iScript reverse transcriptase (iScriptTM Reverse Transcription Supermix for RTqPCR, Bio-Rad, Hercules, CA) as specified by the manufacturer. Quantitative PCR was performed on a CFX96 real-time System (Bio-Rad) using iQ SYBR Green supermix (Bio-Rad). Three standard genes (H2AC: F-CAACGACGAGGAGCTCAACAAG, R-GAAGTTTCCGCAGATTCTGTT GC/ H2AX: F-TCCTGCCCAACATCCAGG, R-TCAGTACTCCTGAGAGGCC TGC/ H1H2BC: F-AACAAGCGCTCGACCATCA, R-GAATTCGCTACGGAGGCTTACT) were used to compute a normalization factor using Genorm software v3.5. The following primer sequences were used to assess muscle denervation: AchRalpha (F-CCACAGACTCAGGGGAGAAG, R-AACGGTGGTGTGTGTTGATG), AchRbeta (F-GGCAAGTTCC TGCTTTTCGG, R-CGTCCGGAAGTGGATGTTCA), AchRdelta (FCGCTGCTTCTGCTTCTAGGG, R-ATCAGTTGGCCTTCGGCTT) and AchRepsilon (F-CAATGCCAATCCAGACACTG, R-CCCTGCTTCTCC TGACACTC).

\subsection{Triple immunofluorescence}

Mice were anesthetized (Ketaset $300 \mathrm{mg} / \mathrm{kg}$ and Domitor $3 \mathrm{mg} / \mathrm{kg}$ by i.p. injection) and intracardially perfused; the tissues were isolated without microdissection and fixed overnight $(\mathrm{O} / \mathrm{N})$ in $4 \%$ PFA at $4{ }^{\circ} \mathrm{C}$. Samples were frozen, cut with a cryostat and kept in cryoprotection solution at $-20{ }^{\circ} \mathrm{C}$ until used.

All immunohistochemistry was done in free-floating $30 \mu \mathrm{m}$ sections from thoracic spinal cord, using usually three stained sections/images per animal. Sections were washed three times for $10 \mathrm{~min}$ each in PBS, blocked (5\% goat serum in $0.3 \%$ Triton X-100/PBS) at room temperature (RT) for $1 \mathrm{~h}$, and incubated with primary antibodies $\mathrm{O} / \mathrm{N}$ at $4{ }^{\circ} \mathrm{C}$. Samples were washed three times in PBS and incubated with corresponding secondary antibodies and DAPI at RT for $1 \mathrm{~h}$. After three 10 min washing steps in PBS, samples were mounted on SuperFrost Plus slides with Mountant PermaFluor mounting medium and stored at $4{ }^{\circ} \mathrm{C}$.

Immunocytochemistry was performed as described (Sen et al., 2019b), blocking with 5\% BSA for $30 \mathrm{~min}$ at RT. Primary antibodies were incubated $\mathrm{O} / \mathrm{N}$ at $4{ }^{\circ} \mathrm{C}$. Images from the different stainings were done with a Nikon Eclipse TE200-E confocal microscope. In all cases, Zstacks were processed with ImageJ software (Schindelin et al., 2012). Photoshop CS5.1 was used to generate figures.

Primary antibodies used were: ATXN2 (BD Transduction 611378, 1:50), IBA1 (Wako 019-19741, 1:1000), PABPC1 (Abcam ab21060, 1:100); TDP43 (Abcam ab41881, 1:100), TIA1 (Santa Cruz sc-1751, $1: 100)$. Secondary antibodies used were: Alexa Fluor 488 goat antimouse IgG A11029, Alexa Fluor 488 goat anti-rabbit IgG A11034, Alexa Fluor 565 rabbit anti-goat IgG A11079, Alexa Fluor 568 donkey anti-sheep IgG A21099, Alexa Fluor 568 goat anti-rabbit IgG A11036 (all Invitrogen, 1:1000).

\subsection{Semiquantitative immunoblots}

Protein extraction from cervicothoracic spinal cord tissue (4 WT vs. 4 KO; 6 WT vs. 5 KIN at 3 months; 7 WT vs. 8 KIN at 14 months) and cells was performed with RIPA buffer as described previously (Sen et al., 2019b). Subsequently, the pellet was re-suspended in SDS lysis buffer (137 mM Tris-HCl pH 6.8, 4\% SDS, 20\% Glycerol, Proteinase inhibitor, Roche), sonicated and quantified using the Pierce BCA Assay Kit (Thermo Fisher Scientific). The following antibodies were used: ACTB (Sigma A5441, 1:10000), ATXN2 (Proteintech 21776-1-AP, 1:500), CASP3 (Cell Signaling 9665, 1:1000), GFAP (Dako ZO334, 1:2000), GPNMB (Biotechne AF 2330, 1:500), IBA1 (Wako 019-19741, 1:2000), NeuN (Millipore ABN78, 1:1000), PGRN (Biotechne AF 2557, 1:250), PQBP1 (Biomol A302-802A-M, 1:500), RIPK1 (Cell Signaling Technology 3493S, 1:500), TDP43 (Abcam ab41881, 1:1000). The secondary antibodies were: IRDye 680RD goat anti-mouse 926-32220, IRDye 800CW donkey anti-goat 926-32214, IRDye 800CW goat anti-mouse 926-32210, IRDye 800CW goat anti-rabbit 926-32211 (all LI-COR, $1: 10000)$.

\subsection{Quantitative reverse-transcriptase PCR in spinal cord}

Total RNA isolation from mouse cervicothoracic spinal cord tissue (4 WT vs. 4 KO; 6 WT vs. 5 KIN at 3 months; 7 WT vs. 8 KIN at 14 months) and RT-qPCR in technical duplicates were done as described previously (Sen et al., 2019b). RNA was quantified with the NanoDrop 1000 spectrophotometer (Thermo Fisher Scientific, Waltham, MA). Tbp was used as the housekeeping gene. The data were analyzed using the $2^{-\Delta \Delta C t}$ method (Schmittgen and Livak, 2008). TaqMan assays from Thermo Scientific were used for Aif1 Mm00479862_g1, Ano3 Mm01270409_m1, Atxn2 Mm01199902_m1, C1qa Mm00432142_m1, C1qb Mm01179619 _m1, C1qc Mm00776126_m1, C3 Mm01232779_m1, Calb2 Mm00801461_m1, Casp3 Mm01195085_m1, Cdr1 Mm04178856_s1, Cirbp Mm00483336_g1, Clgn Mm00515526_m1, Cybb Mm01287743_ m1, Cyp46a1 Mm00487306_m1, Cyp51a1 Mm00490968_m1, Dcp1a 
Mm00460131_m1, Dcp1b Mm01183995-m1, Dcp2 Mm01264061_m1, Dcps Mm00510029_m1, Ddx1 Mm00506205_m1, Ddx6 Mm006193 26_m1, Dhcr24 Mm00519071_m1, Dhx15 Mm00492113_m1, Eif5a2 Mm00812570_g1, Elovl5 Mm00506717_m1, Fmr1 Mm01339582_m1, Gfap Mm01253033_m1, Gnal Mm01258217_m1, Gpnmb Mm013 28587_m1, Grn Mm00433848-m1, Hecw1 Mm00462346_m1, Hmgcs1 Mm01304569_m1, Hnrnpa2b1 Mm01332941_m1, Hnrnpd Mm01201314_m1, Idi1 Mm01337454_m1, Irak4 Mm00459443_m1, Kcna1 Mm00439977_s1, Kcna2 Mm00434584_s1, Kif5a Mm005152 65_m1, Kif5b Mm01225510_g1, Kif5c Mm00500464_m1, Lsm1 Mm01600253_m1, Msmo1 Mm00499390_m1, Parva Mm00480444_m1, Pcbp1 Mm00478712_s1, Pcbp2 Mm01296174_g1, Pcbp3 Mm011497 50_m1, Pcbp4 Mm00451991_g1, Prpf19 Mm01208295_m1, Ptbp1 Mm01731480_gH, Pura Mm01158049_s1, Puf60 Mm00505017_m1, Rbfox3 Mm01248771_m1, Ripk1 Mm00436354_m1, Rnaset2a/b Mm02601904_m1, Scn4b Mm01175562_m1, Srrm2 Mm00613771_m1, Tacr1 Mm00436892_m1, Tardbp Mm00523866_m1, Tbp Mm00446 973_m1, Tlr3 Mm01207404_m1, Tlr7 Mm00446590_m1, Tlr9 Mm00446193_m1, Trem2 Mm00451744_m1, Ttbk2 Mm00453709_m1, Tyrobp Mm00449152_m1, Uhmk1 Mm00495171_m1, Unc80 Mm0061 5703_m1, Ybx1 Mm00850878_g1.

\subsection{BV2 microglia cell line culture}

Murine microglia cell line BV2 (Blasi et al., 1990) was cultured in DMEM supplemented with $10 \%$ FBS, 1x L-glutamine and 1x Pen/Strep. BV2 cells were seeded at $5 \times 10^{4}$ cells/well on poly-D-lysine $(0.1 \mathrm{mg} / \mathrm{mL})$ coated glass slides in a 12-well plate $\mathrm{O} / \mathrm{N}$ and then were stressed with 0.25 mM Sodium Arsenite (NaArs, S7400-100G Sigma-Aldrich) for 15 min. Cells were fixed with 4\% PFA at RT for $20 \mathrm{~min}$, permeabilized with $0.1 \%$ Triton X-100/PBS for 20 min and washed three times with DPBS before staining.

\subsection{Global transcriptomics by Clariom D microarrays}

Cervicothoracic spinal cord RNA integrity was controlled by the 2100 Bioanalyzer with the Nano Assay (Agilent Technologies, Santa Clara, CA). As recommended, $1 \mu \mathrm{g}$ of RNA was pre-treated with DNase amplification grade (Invitrogen). The GeneChip ${ }^{\mathrm{TM}}$ WT PLUS Reagent Kit (Applied Biosystems, Foster City, CA) was used to generate singlestranded cDNA (ss-cDNA) following the manufacturer's instructions. The ss-cDNA was fragmented and labeled right before the hybridization to a Clariom D Array (Thermo Fisher Scientific). The arrays were scanned with the Affymetrix GeneChip Scanner and the data were processed with the Transcriptome Analysis Console (TAC) 4.0.1 (Applied Biosystems) using default algorithm parameters.

\subsection{STRING protein-protein interaction bioinformatics} 11.0 .

The web-server https://string-db.org/ was used in 2019 with version

\subsection{Quantification of cholesterol pathway intermediates by gas chromatography mass spectrometry using selective ion detection methodology}

Wet tissue specimens of cervicothoracic spinal cord without perfusion were dried for about $24 \mathrm{~h}$ in a Savant SpeedVac DNA 120 Concentrator (Thermo Scientific, Langenselbold, Germany). Exactly 1 $\mathrm{ml}$ of a mixture of chloroform/methanol (2:1) was added to about $10 \mathrm{mg}$ of the dried specimen and the lipids were extracted for $12 \mathrm{~h}$. After centrifugation, the supernatant was completely transferred into a $10 \mathrm{~mL}$ glass tube, $50 \mu \mathrm{g} 5 \alpha$-cholestane (Serva, Heidelberg, Germany) ( $50 \mu \mathrm{l}$ from a stock solution of $5 \alpha$-cholestane in cyclohexane [Merck KGaA, Darmstadt, Germany]; $1 \mathrm{mg} / \mathrm{ml}$ ), $1 \mu \mathrm{g}$ epicoprostanol (Sigma, Deisenhofen, Germany) (10 $\mu \mathrm{l}$ from a stock solution epicoprostanol in cyclohexane; $100 \mu \mathrm{g} / \mathrm{ml})$ and $50 \mathrm{ng}$ racemic $\left[23,23,24,25-{ }^{2} \mathrm{H}_{4}\right] 24(\mathrm{R}, \mathrm{S})-$ hydroxycholesterol (Medical Isotopes Inc., Pelham, NH, USA), $100 \mathrm{ng}$ 26.26.26.27.27.27- $\left[{ }^{2} \mathrm{H}_{6}\right]-7$ alpha-hydroxycholesterol, and $100 \mathrm{ng}$ [2.2.3.4.6- $\left.{ }^{2} \mathrm{H}_{5}\right]-(25 \mathrm{R}) 27-$ hydroxycholesterol (Medical Isotopes Inc. Pelham, NH, USA) $(50 \mu \mathrm{l}$ from a stock solution in toluene [Merck KGaA, Darmstadt, Germany]; $2 \mu \mathrm{g} / \mathrm{ml}$ ), respectively, were added as internal standards. To avoid autoxidation, $50 \mu \mathrm{l}$ of a 2.6.-di-tert.-butylmethylphenol/methanol solution ( $\mathrm{mg} / \mathrm{mL}$ ) (Sigma-Aldrich Chemie $\mathrm{GmbH}$, Taufkirchen, Germany) was added additionally.

Characteristic sterols and oxysterols were quantified in order to describe cholesterol metabolism in the given tissues. The O-trimethylsilylated sterol and di-trimethylsilylated oxysterol ethers were separated by gas chromatography from the same lipid liver extract in analogy to (Mackay et al., 2014; Sosic-Jurjevic et al., 2019; Thelen et al., 2006).

Cholesterol was detected by less sensitive flame-ionization detection (FID) ( $5 \alpha$-cholestane, internal standard, ISTD), the non-cholesterol sterols (epicoprostanol, ISTD) and the oxysterols $\left({ }^{2} \mathrm{H}_{\mathrm{x}}\right.$-oxysterols, ISTD) by highly specific and sensitive mass spectrometry in the selected ion monitoring mode (MS-SIM). Gas chromatographic separation and detection of cholesterol and 5alpha-cholestane (ISTD) was performed on a DB-XLB $30 \mathrm{~m}$ x $0.25 \mathrm{~mm}$ i.d. x $0.25 \mu \mathrm{m}$ film thickness (J\&W Scientific Alltech, Folsom, CA, U.S.A.) in an Agilent Technologies 7890B GC System (Agilent Technologies, Palo Alto, CA, U.S.A), equipped with an FID. The GC-FID instrument is controlled by a computer with the Agilent OpenLAB CDS Software version C.01.07. Authentic sterols and deuterium labeled oxysterols were separated on another DB-XLB column (30 $\mathrm{m} \times 0.25 \mathrm{~mm}$ i.d. $\times 0.25 \mu \mathrm{m}$ film thickness, J\&W Scientific Alltech, Folsom, CA, U.S.A.) in a Agilent Technologies 7890B GC System (Agilent Technologies, Waldbronn, Germany) connected with a direct capillary inlet system to a quadrupole mass selective detector 5977B inert MSD (Agilent Technologies, Waldbronn, Germany). Both GC systems were equipped with Agilent Technologies 7693 series autosamplers and injector plus trays. The GC-MSD instrument is controlled by a computer with the Agilent MS MassHunter Software version B.07.05.

After saponification with $2 \mathrm{~mL} 1 \mathrm{M} 95 \%$ ethanolic sodium hydroxide solution (Merck KGaA, Darmstadt, Germany) at $60{ }^{\circ} \mathrm{C}$ for one hour, the free sterols and oxysterols were extracted three times with $3 \mathrm{~mL}$ cyclohexane each. The organic solvent was evaporated by a gentle stream of nitrogen at $60{ }^{\circ} \mathrm{C}$ on a heating block. The residue was dissolved in $80 \mu \mathrm{L}$ n-decane (Merck KGaA, Darmstadt, Germany). An aliquot of $40 \mu \mathrm{L}$ was incubated $\left(1 \mathrm{~h}\right.$ at $70{ }^{\circ} \mathrm{C}$ on a heating block) by addition of $20 \mu \mathrm{L}$ of the trimethylsilylating (TMSi) reagent (chlortrimethylsilane [Merck KGaA, Darmstadt, Germany]/1.1.1.3.3.3-Hexamethyldisilasane [Sigma Aldrich, Co., St. Louis, MO, U.S.A ]/pyridine [Merck KGaA, Darmstadt, Germany], 9:3:1) in a GC vial for GC-MSD non-cholesterol and oxysterol analysis. Another aliquot of $40 \mu \mathrm{L}$ was incubated by addition of $40 \mu \mathrm{L}$ of the TMSi-reagent and dilution with $300 \mu \mathrm{L}$-decane in a GC vial for GCFID cholesterol analysis.

An aliquot of $2 \mu \mathrm{l}$ was injected by automated injection in a splitless mode using helium (1 $\mathrm{mL} / \mathrm{min})$ as carrier gas for GC-MS-SIM and hydrogen $(1 \mathrm{ml} / \mathrm{min})$ for GC-FID analysis at an injection temperature of $280^{\circ} \mathrm{C}$. The temperature program for GC was as follows: $150{ }^{\circ} \mathrm{C}$ for three minutes, followed by $20^{\circ} \mathrm{C} / \mathrm{min}$ up to $290^{\circ} \mathrm{C}$ keeping for 34 minutes. For MSD electron impact ionization was applied with $70 \mathrm{eV}$. SIM was performed by cycling the quadrupole mass filter between different $\mathrm{m} / \mathrm{z}$ at a rate of 3.7 cycles/sec. Non-cholesterol sterols were monitored as their TMSi-, the oxysterols as their di-TMSi-derivatives using the following masses: epicoprostanol $\mathrm{m} / \mathrm{z} 370\left(\mathrm{M}^{+}-90, \mathrm{M}^{+}\right.$-OTMSi), lathosterol at $\mathrm{m} / \mathrm{z} 458\left(\mathrm{M}^{+}\right)$, desmosterol at $\mathrm{m} / \mathrm{z} 441\left(\mathrm{M}^{+}-15, \mathrm{M}^{+}-\mathrm{CH}_{3}\right)$, lanosterol at $\mathrm{m} / \mathrm{z} 393\left(\mathrm{M}^{+}-90-15, \mathrm{M}^{+}\right.$-OTMSi- $\left.\mathrm{CH}_{3}\right)$, 24.25-dihydrolanosterol at $\mathrm{m} / \mathrm{z} 395\left(\mathrm{M}^{+}-90-15, \mathrm{M}^{+}-\mathrm{OTMSi}-\mathrm{CH}_{3}\right)$, cholestanol at $\mathrm{m} / \mathrm{z} 306$, 26.26.26.27.27.27- $\left[{ }^{2} \mathrm{H}_{6}\right]-7$ alpha-hydroxycholesterol at $\mathrm{m} / \mathrm{z} 462\left(\mathrm{M}^{+}\right.$90), 7alpha-hydroxycholesterol at $\mathrm{m} / \mathrm{z} 456\left(\mathrm{M}^{+}-90\right),\left[23,23,24,25-{ }^{2} \mathrm{H}_{4}\right]$ 24(R,S)-hydroxycholesterol at $\mathrm{m} / \mathrm{z} 416\left(\mathrm{M}^{+}-90-44, \mathrm{M}^{+}\right.$-OTMSi-CD $\left.\left(\mathrm{CH}_{3}\right)_{2}\right), 24(\mathrm{~S})$-hydroxycholesterol at $\mathrm{m} / \mathrm{z} 413\left(\mathrm{M}^{+}-90-43, \mathrm{M}^{+}\right.$-OTMSi- 
$\mathrm{CH}\left(\mathrm{CH}_{3}\right)_{2},\left[2,2,3,4,6-{ }^{2} \mathrm{H}_{5}\right]-(25 \mathrm{R}) 27-$ hydroxycholesterol at $461\left(\mathrm{M}^{+}-90\right)$, (25R)27-hydroxycholesterol at $456\left(\mathrm{M}^{+}-90\right)$. Peak integration was performed manually. Cholesterol was directly quantified by multiplying the ratios of the area under the curve of cholesterol to 5alpha-cholestane by $50 \mu \mathrm{g}$ (ISTD amount). Non-cholesterol sterols and oxysterols were quantified from the ratios of the areas under the curve of the respective non-cholesterol sterols/oxysterol after SIM analyses against internal standards using standard curves for the listed sterols/oxysterols. Identity of all sterols was proven by comparison with the full-scan mass spectra of authentic compounds. Additional qualifiers (characteristic fragment ions) were used for structural identification ( $\mathrm{m} / \mathrm{z}$ values not shown).
A

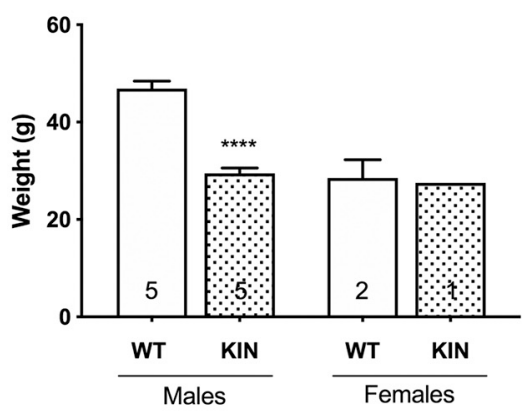

B
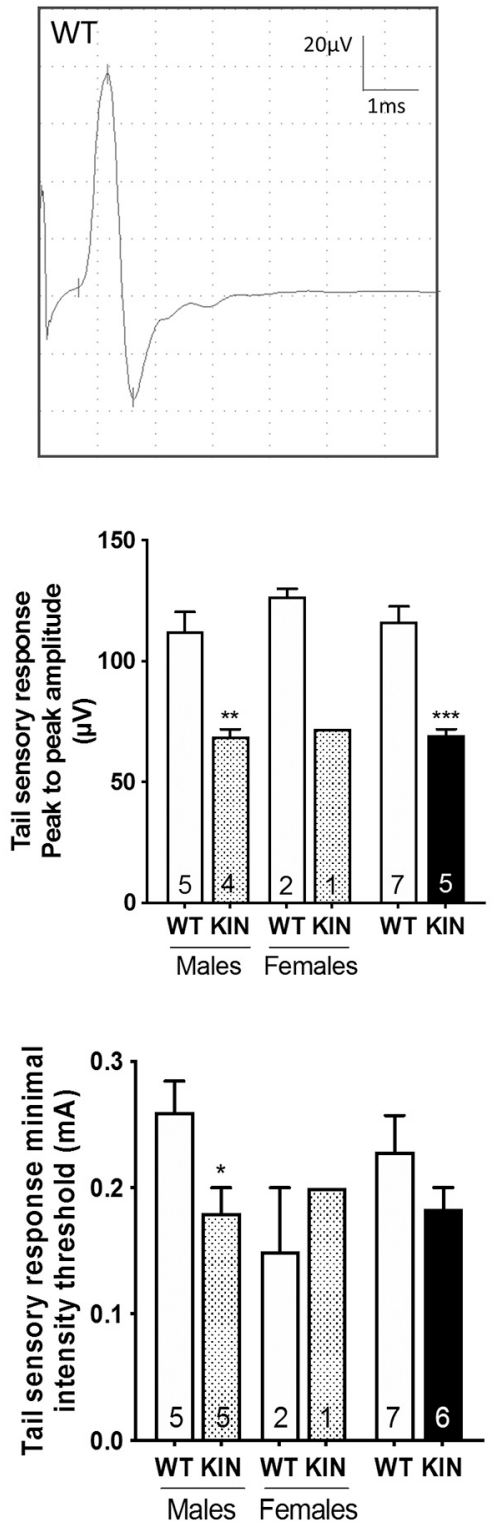
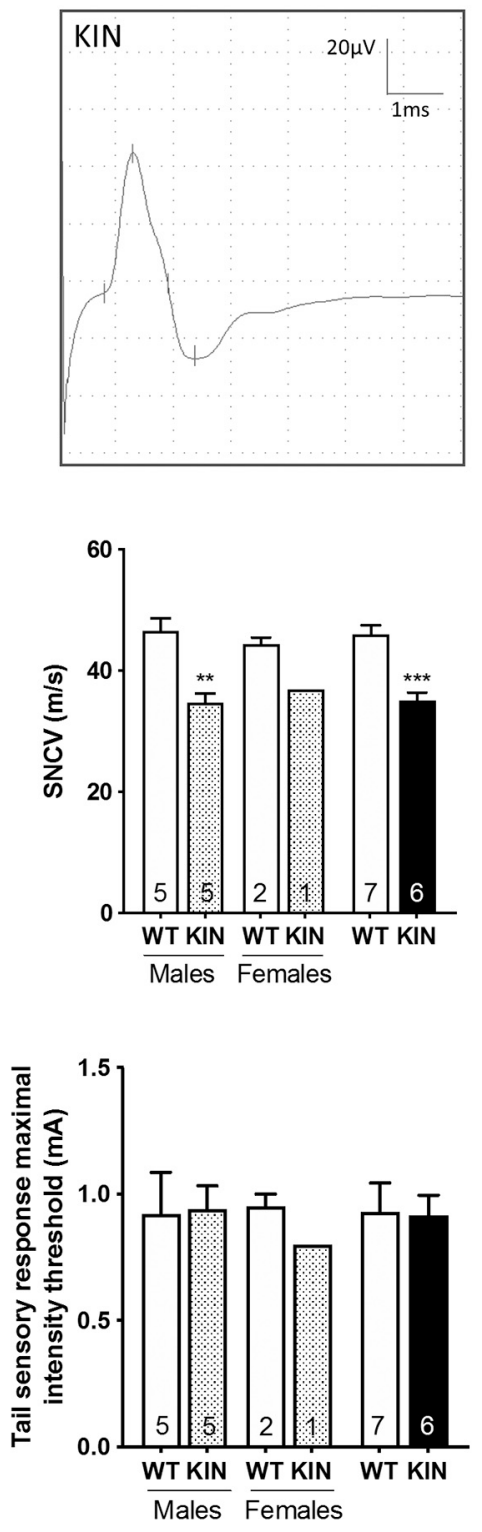

Fig. 1. Functional assessment of hindlimb nerve function detects early sensory, predominantly axonal neuropathy. Assessment of 5 male and 1 female Atxn2-CAG100-KnockIn (KIN) mice with age-/sex-matched wildtype (WT) controls at the age of 9-10 months (animal numbers per subgroup indicated within bar graphs, separately showing each gender and the sum of both genders) confirmed the previously published (A) weight reduction with significance for the male mutants. (B) Analyses of sensory nerve action potentials (SNAP) demonstrated peak to peak amplitude and sensory nerve conduction velocity (SNCV) from tail to be reduced and the perception of minimal intensity stimuli to be affected. Top: representative SNAP recordings; sensitivity $20 \mu \mathrm{V} / \mathrm{div}$, sweep speed $1 \mathrm{~ms} /$ div. Middle: Peak to peak amplitude (left) and SNCV (right). Bottom: minimal (left) and maximal (right) intensity threshold. After assessing normal distribution, unpaired Student's $\mathrm{t}$ test was employed, significances were illustrated with asterisks ( ${ }^{*} p<0.05$; ${ }^{* *} p<0.005$; ${ }^{* * *} p<0.0001$ ), values represent the means \pm SEM. 


\subsection{Statistics and graphical visualization}

Statistical tests were performed as unpaired Student's t-test with Welch's correction using GraphPad Prism software version 7.02. Figures display the mean and standard error of the mean (SEM) values. Significance was assumed at $\mathrm{p}<0.05$ and highlighted with asterisks: ${ }^{*} p<0.05, * * p<0.01, * * * p<0.001, * * * * p<0.0001$.

\section{Results}

\subsection{Neurophysiology of lower limbs finds mainly axonal sensory} neuropathy as earliest deficit

The initial characterization of our novel Atxn2-CAG100-KnockIn (KIN) mouse mutants had demonstrated a peripheral motor weakness upon forelimb grip strength analysis in comparison to sex-/age-matched wildtype (WT) controls from the age of 11 months onwards (Sen et al., 2019b). To elucidate if motor neuron denervation and sensory neuropathy are early features within the locomotor phenotype of this SCA2 model as in patients, neurophysiological analyses by hindlimb electroneurography (ENG) were now performed at the age of 9-10 months, when a weight reduction due to the toxic ATXN2 gain-of-function is already evident in males, as previously reported (Sen et al., 2019b). Gender differences of phenotype have been observed also in other mouse models of ALS (Kreilaus et al., 2020), so we separated the results by sex. Sensory potentials showed significant reductions of amplitude (to $\sim 60 \%$ ), conduction velocity (SNCV, to $\sim 80 \%$ ), and, for males, minimal intensity threshold (to $\sim 70 \%$ ) (Fig. 1). In contrast, for the compound motor action potentials (CMAP) there was no significant change of velocity, amplitude, minimal and maximal intensity threshold at this disease stage. Upon electromyography (EMG) routines, no muscle denervation potentials such as fasciculations or fibrillations were detected (Suppl. Fig. S1A). The reverse-transcriptase quantitative realtime polymerase chain reaction (RT-qPCR) analysis of Acetylcholine Receptor (AchR) alpha/beta/delta/epsilon subunit mRNA expression in the Tibialis Anterior and Soleus muscle homogenates showed no significant reduction (Suppl. Fig. S1B). These observations indicate a loss of some fast-conducting axons and of myelin wrapping that reduce the perception of slight stimuli as initial dysfunction in peripheral nerves, before the advent of later motor deficits. In SCA2 patients, somatosensory denervation of lower limbs is indeed an early phenomenon (Rub et al., 2007; Velazquez-Perez et al., 2014), while the motor neuron pathology at disease onset affects primarily the face, neck and arms (Estrada et al., 1999; Medrano-Montero et al., 2018). We made no further efforts to characterize the preferential sensory affection by histology in the KIN mouse, since our previous analyses of SCA2 patient autopsies had already documented widespread degeneration from dorsal root ganglion via ascending tracts and nuclei until thalamus (Estrada et al., 1999; Rub et al., 2003b). Overall, these data demonstrate sensory neuropathy, but not yet motor denervation of KIN hindlimbs at the age of 9-10 months.

\subsection{Progressive sequestration of PABPC1 together with nuclear TDP43 and TIA1 proteins into cytosolic ATXN2 aggregates in spinal motor neurons}

To further investigate the reduced grip strength in the Atxn2CAG100-KIN mice from the age of 11 months onwards, the spinal cord pathology was examined by histology. Previous analyses of cerebellar Purkinje neurons in 2-year-old mice with KnockIn of an Atxn2-CAG42expansion, a frequent size among SCA2 patients that triggers clinical onset at ages around 30 years, demonstrated a pathological aggregation process where cytosolic ATXN2 sequestrates its direct interactor protein PABPC1 [poly(A)-binding-protein-cytoplasmic-1] into insolubility (Damrath et al., 2012; Sen et al., 2019b). In an effort to test this observation in our new Atxn2-CAG100-KIN mice for spinal cord motor neurons, triple immunofluorescence was employed to study animals at the early adult age of 3 months, at the age of initial weight deficits around 6 months, and the prefinal age of 14 months. As shown in Fig. 2, the yellow-stained colocalization of green ATXN2 signals and red PABPC1 signals within cytosolic clumps were clearly visible at 6 months and became more numerous by 14 months. Additional detection of nuclear TDP43, which is known to interact with ATXN2 indirectly via RNA-association within short-lived stress granules (Elden et al., 2010; Zhang et al., 2018), also demonstrated colocalization in the cytosolic ATXN2 aggregates, albeit only at later age to a massive degree (Fig. 2). This observation provides evidence of progressive spinal motor neuron affection leading to a motor neuropathy in this SCA2 mouse model over time. A similar pathological relocalization from a nuclear position to the cytosolic aggregates was also observed for the RNA-binding protein TIA1 (Suppl. Fig. S2), an established marker of stress granules (Fitzgerald and Semler, 2013). As a neuropathological diagnostic hallmark of spinal motor neuron affection in ALS, the nuclear depletion and cytoplasmic accumulation of TDP43, TIA1 and other RNA-binding proteins is probably reflecting a crucial disease mechanism (Chen-Plotkin et al., 2010; Kim and Taylor, 2017; Li et al., 2013). The TDP43 mislocalization to the cytosol was already reported for SCA2 patients in lower and upper motor neurons, cerebellar Purkinje cells, brainstem neurons and other neuron populations (Baumer et al., 2014; Elden et al., 2010; Toyoshima et al., 2011), so the animal model provides a faithful reflection of the temporal development and the spatial distribution of the human pathology.

\subsection{Cascade of molecular events upstream from TDP43 mislocalization is mirrored in spinal cord}

The importance of TDP43 mislocalization for ALS and FTLD has prompted numerous studies into the underlying molecular events, so we investigated whether the pathomechanisms upstream from TDP43 aggregation can also be found in the Atxn2-CAG100-KIN spinal cord homogenates. It was reported that ATXN2 expansions trigger stressdependent activation of caspase-3 (CASP3), which is responsible for the proteolytic C-terminal cleavage and cytoplasmic retention of TDP43 (Hart and Gitler, 2012). To test whether this pathogenesis feature is present, and if it is dependent (i) on the physiological function of ATXN2, (ii) on the polyQ expansion or (iii) on disease progression, we studied cervicothoracic spinal cord from mice with Atxn2-KO versus Atxn2-CAG100-KIN at the presymptomatic age of 3 months versus the preterminal age of 14 months. Indeed, semiquantitative immunoblots demonstrated an elevated abundance of TDP43 (1.6-fold) and CASP3 protein (1.8-fold) only in KIN mice at the old age, while their mRNA retained normal expression (Fig. 3A/B). Given that the immunoblots were done in RIPA extracts and therefore are unlikely to represent aggregated proteins, the selective modulation of protein abundance but not mRNA expression might be due to enhanced mRNA translation due to a gain in the physiological function of ATXN2 at ribosomes. The elevated abundance of both proteins occurs only in polyQ expansion tissue during the progressive aggregation process, and may represent two driving forces for the formation of TDP43 deposits in the cytosol. At the age of 14 months the neuronal mass appeared already reduced below $>0.6$-fold (as estimated via semiquantitative immunoblots of the marker NeuN, Fig. 3A), in parallel to a similar 2-fold increase of astrogliosis (marker GFAP, Fig. 3A and B) and together with substantial microgliosis (marker IBA1, encoded by Aif1 mRNA, Fig. 3B).

Furthermore, mutations of progranulin (PGRN, encoded by $\mathrm{Gr}$ mRNA) were demonstrated in FTLD individuals to cause cytoplasmic TDP43 mislocalization (Rademakers et al., 2012). The depletion of progranulin also leads to accumulation of TDP43 fragments, in a caspase-dependent manner (Zhang et al., 2007). Intracellular PGRN gives rise to processed secreted isoforms. They stabilize the protease CTSD in lysosomes and extracellularly to stimulate axonal outgrowth (Beel et al., 2017). The absence of PGRN conversely acts as trigger of 


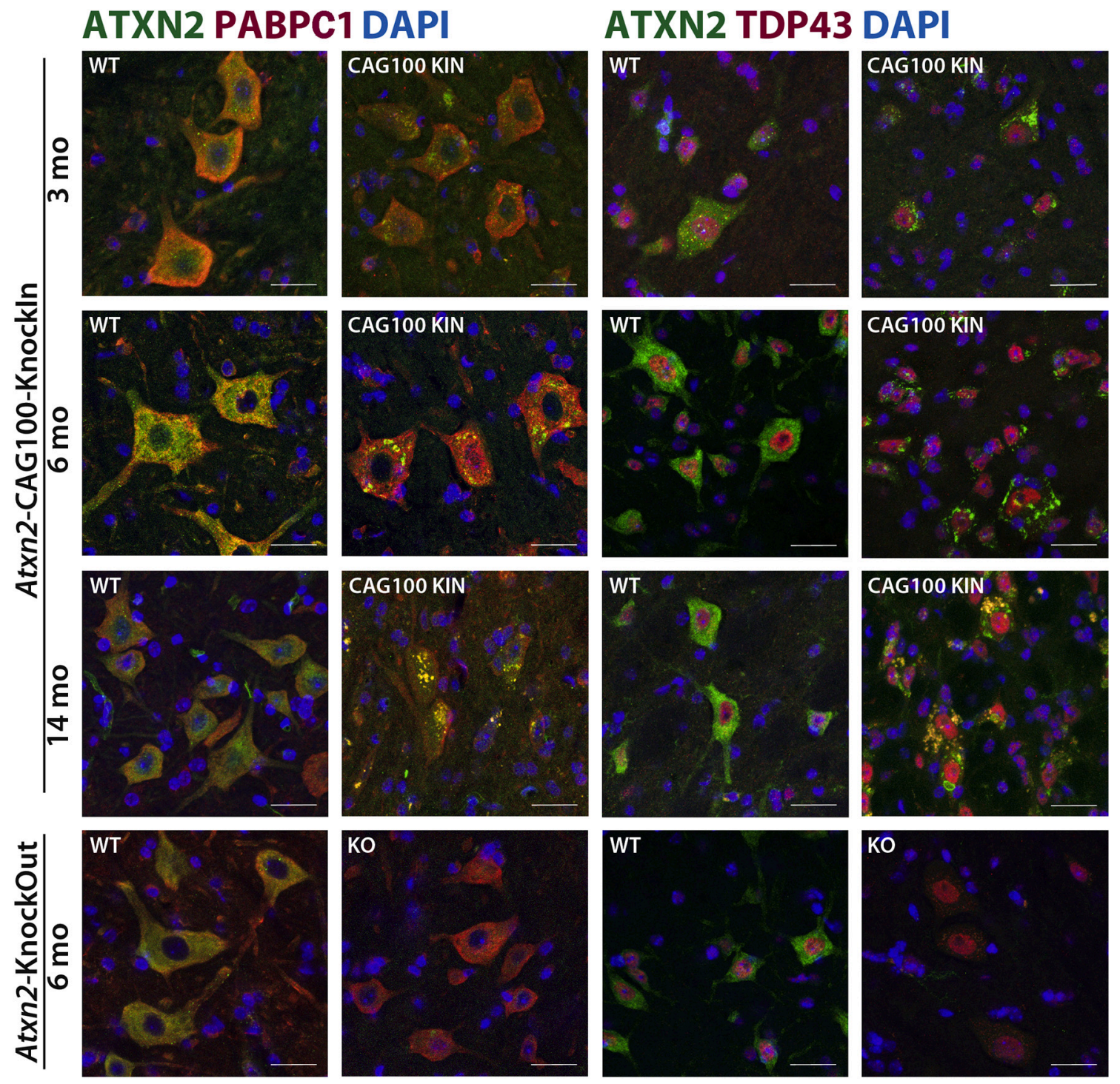

Fig. 2. ATXN2 protein aggregates in spinal motor neurons progressively sequestrate PABPC1 and TDP43. Triple co-immunofluorescent staining of ATXN2 (green) with PABPC1 or TDP43 (red) in Atxn2-CAG100-KIN (at 3, 6 or 14 months of age) versus Atxn2-KO mice (at 6 months) versus age-/sex-matched WT controls (3 stained sections from 1 animal per time point). The sequestration process co-localizes both proteins in cytosolic foci and produces a yellow signal in the merged panels. Nuclei were detected by DAPI (blue color), scale bar reflects $25 \mu \mathrm{m}$.

synaptic pruning by microglia via the complement membrane attack complex (Lui et al., 2016). It was therefore interesting to observe significantly increased Grn mRNA levels in 14-month-old KIN spinal cord (Fig. 3C/D) as a quite specific response to the TDP43 pathology, possibly representing a compensatory effort.

PGRN abundance is modulated in response to RIPK1-mediated phosphorylation (Mason et al., 2017), whose activation can sense viral or toxic nucleotides via the toll-like receptor TLR3 (Kuriakose and Kanneganti, 2018; Newton, 2019), drives pro-inflammatory necroptosis (Yuan et al., 2019), and is implicated in ALS (Ito et al., 2016; Xu et al., 2018). Consistent with these reports, enhanced RIPK1 protein levels were observed in the spinal cord of Atxn2-CAG100-KIN mice, possibly with a 1.4-fold abundance already at 3 months, but achieving significance only for the 1.5-fold increase at the age of 14 months (Fig. 3C/D). The Ripk1 mRNA levels showed a clearly significant increase to almost 2fold at the age of 14 months.

Toll-like-receptor (TLR)-triggered activation of microglial cells, as well as inflammatory lysis of synapses via neuronal deposition of complement C1q and C3 factors on their membranes, were carefully documented in ALS spinal cord tissue (Casula et al., 2011; Parker et al.,
2019). Again, in the 14-month-old spinal cord of Atxn2-CAG100-KIN mice there were strong transcriptional inductions for Tlr3, Tlr7, Tlr9, $C 1 q a, C 1 q b, C 1 q c$ and $C 3$ (Suppl. Fig. S3A). The particularly strong induction of Tlr7 as sensor (at endosomes or lysosomes) for toxic singlestranded RNA and miRNA (3.5-fold), in comparison to Tlr9 as sensor of single-stranded DNA sequences (2.3-fold) and Tlr3 as sensor of double-stranded RNA (1.3-fold) underlined and elucidated the RNAtoxicity (Hartmann, 2017; Heneka et al., 2014; Lehmann et al., 2012) present in the spinal cord tissue of our SCA2 model. It is interesting to note that Ataxin-2 domains were reported to play a role in the miRNAmediated mRNA degradation (Kozlov et al., 2010) and to interact with the microRNA effector DDX6 to mediate quality control of mRNAs (Ariumi et al., 2011; McCann et al., 2011; Nonhoff et al., 2007; Sudhakaran et al., 2014).

Finally, it is known that non-expanded polyQ-domain containing proteins are being recruited into the aggregates formed by expanded ATXN2 or expanded ATXN3 (Perez et al., 1998; Uchihara et al., 2001). Other proteins also bind specifically to expanded polyQ domains, e.g. PQBP1 (Polyglutamine binding protein 1) as a sensor of viral or toxic nucleotides within the innate immunity pathway of brain cells (Waragai 
A
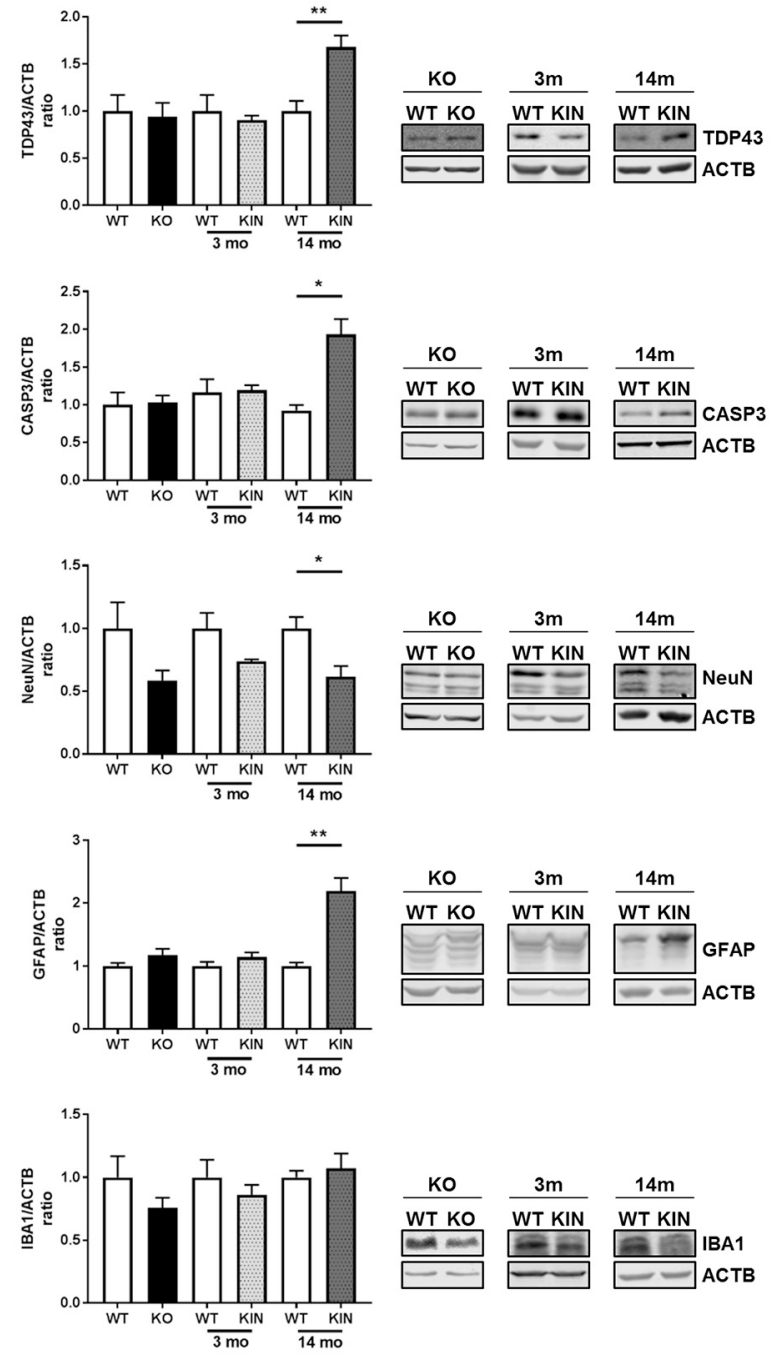

C
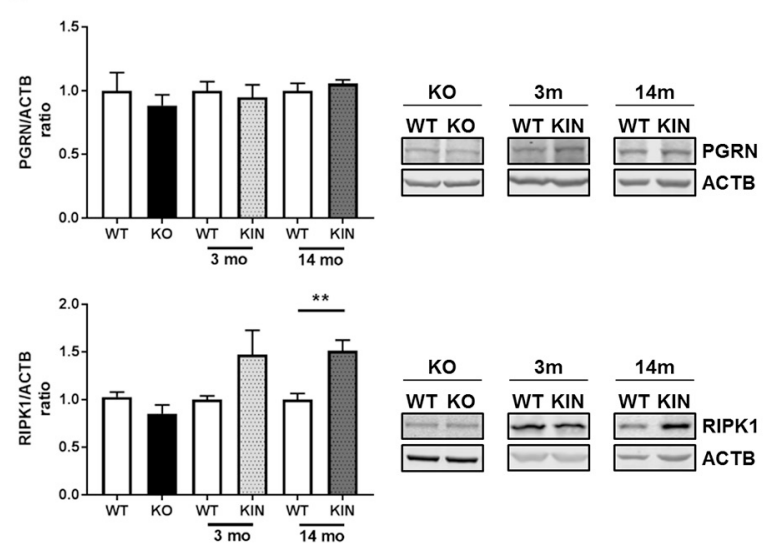

B
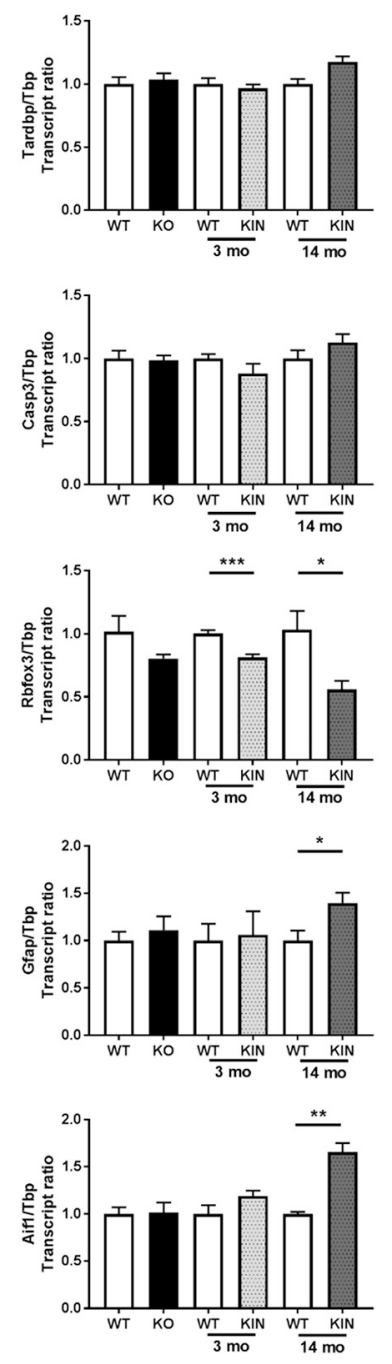

D
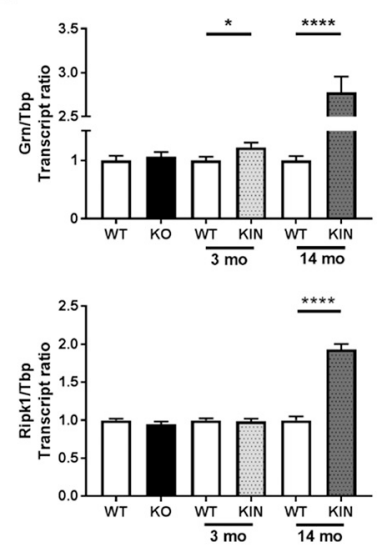

Fig. 3. Validation of protein and mRNA level dysregulations. (A) Semiquantitative immunoblots from RIPA extracts confirmed neuronal loss (marker NeuN), astrogliosis (marker GFAP) and microgliosis (marker IBA1) to occur in Atxn2-CAG100-KIN spinal cord at the preterminal stage of 14 months age, but not at the early KIN stage of 3 months age and in the Atxn2-KO at 6 months. Significantly increased levels in KIN at 14 months were also shown for TDP43 and the factor responsible for its cleavage, CASP3. (B) Quantitative RT-PCR analyses showed a significant deficit of NeuN transcript (Rbfox3) already at incipient disease stage in 3-month-old KIN, whereas astrogliosis (marker Gfap) and microgliosis (marker IBA1 transcript Aif1) became significant at late state. Protein abundance (C) and transcript levels (D) were also documented for PGRN (encoded by Grn mRNA) as molecular marker of lysosomal activation and atrophy, as well as RIPK1 as molecular marker of RNAtoxicity and necroptosis. Again, a significant elevation of Grn mRNA at the age of 3 months suggested atrophy and lysosomal breakdown to occur in parallel with first locomotor deficits, predating necroptotic cell death. Immunoblots and RT-qPCR were performed in 4 WT vs. 4 KO at 6 months; 6 WT vs. 5 KIN at 3 months; 7 WT vs. $8 \mathrm{KIN}$ at 14 months. Mean and SEM values are shown, illustrating significance upon Student's t-test with Welch's correction via asterisks: $\mathrm{p}<0.05 *, \mathrm{p}<0.01 * *, \mathrm{p}<0.001 * * *, \mathrm{p}<0.0001 * * * *$. et al., 1999; Yoh et al., 2015). Indeed, in 14-month-old Atxn2-CAG100KIN spinal cord a significant accumulation was documented (Suppl. Fig. S3B). Overexpression of PQBP1 in transgenic mice results in a loss of spinal motor neurons as well as a loss of cerebellar Purkinje neurons (Okuda et al., 2003). Deletion mutations in PQBP1 affect the turnover of FMRP (fragile-X mental retardation protein) in neuronal RNA granules and trigger synaptic dysfunction (Zhang et al., 2017).

Jointly, these analyses of protein abundance or mRNA expression demonstrate a cascade of abnormal molecular events, where interactions of toxic RNAs affect endosomal TLRs and cytosolic PQBP1, which act as pathology sensors together with RIPK1, affecting PGRNdependent neurite growth and CASP3-modulated TDP43 aggregation. This molecular cascade was defined for the motor neuron pathology in ALS and it is observed also in the spinal cord of our SCA2 model at advanced stages. 
3.4. Activated microglia contains ATXN2 aggregates and is mainly proinflammatory, possibly also by cell-autonomous affection

Upon close inspection of spinal cord immunohistochemistry, in the 14-month-old Atxn2-CAG100-KIN mice some ATXN2 aggregates appeared positioned outside motor neurons. Triple immunofluorescence staining showed them to colocalize with the microglia marker IBA1 and these microglia cells showed pronounced activation with larger cell size and thicker branches (Fig. 4A). Within their cytoplasm, beyond the ATXN2 aggregates also a diffuse ATXN2 staining was visible, compatible with the known stress-dependent induction of Ataxin-2 expression (Lastres-Becker et al., 2016). Indeed, publically available RNAseq findings in diverse brain cell populations from murine and human samples (http://brainrnaseq.org/) documented Ataxin-2 transcript expression to be similar in neurons and astrocytes, while Ataxin-2 transcript expression in oligodendrocytic, microglial and endothelial cells ranges at 30$50 \%$ in comparison to neurons. To assess the presence of ATXN2 within microglia at the protein level, the microglial cell line BV2 was immunocytochemically stained for ATXN2 and its direct interactor protein PABPC1, while exposure to oxidative stress via NaArs administration was used to trigger the formation of characteristic stress granules, with the relocalization of ATXN2 and PABPC1 there ensuring staining specificity. The immunofluorescent signals confirmed the typical diffuse cytosolic pattern for both proteins that changes to multiple granules with the expected delay (Fig. 4B). These data indicate that microglia would not only phagocytose ATXN2 aggregates that were produced by neurons, but may also suffer in cell-autonomous manner from the RNA toxicity triggered by the ATXN2 mutation. To address the question whether the activated microglia cells in old KIN spinal cord are antiinflammatory protective (M2 differentiation) or rather show the proinflammatory toxic properties (M1 differentiation) that were documented in ALS (Geloso et al., 2017), expression of the phagocytosis factor TREM2 and its adaptor DAP12 (encoded by Tyrobp) as M2 markers, as well as the common downstream TLR signaling element IRAK4 and the respiratory burst oxidase subunit NOX2 (encoded by $C y b b$ mRNA) as M1 marker (Cameron et al., 2012; Jiang et al., 2018; Pena-Altamira et al., 2016; Zhao et al., 2015) was quantified by RTqPCR. While Trem2 and Tyrobp showed about 3-fold induction and Irak4 exhibited 1.5-fold induction, the superoxide-production enzyme $C y b b$ was elevated 7-fold (Suppl. Fig. S4), indicating a massive proinflammatory M1 activation of microglial cells in parallel to a still substantial M2 activation, as previously observed in traumatic brain injury (Morganti et al., 2016; Ransohoff, 2016). These data further confirm that microglia are not acting within physiological compensation efforts, but show abnormal profiles that may reflect cell-autonomous pathology. In view of recent biochemical reports that ATXN2 and its sequence homologous interactor protein ATXN2L are associated in the cytosol with immune modulators like PKR, MNDA and TNIP2 (Banks et al., 2016; Diner et al., 2015; Varjosalo et al., 2013), the neuroinflammatory phenotype of our KIN mice may be an important contributor to pathology.

Altogether, the strong microglial activation in spinal cord at prefinal stages does not only serve neuroprotective functions via ATXN2 aggregate phagocytosis, but shows prominent toxic pro-inflammatory features, possibly via cell-autonomous sensitization of microglia to RNA toxicity caused by the stress-augmented expression of expanded ATXN2.

\subsection{Expression profiles of spinal cord at incipient versus preterminal} disease stage consistently show increases for RNA-binding proteins and bioenergetics markers, decreases for tyrosine kinase signaling factors, synapse/axon components, and cholesterol biosynthesis enzymes

In view of the crucial role of ATXN2 and stress granules for RNA quality control, we surveyed the global transcriptome with Clariom D microarrays containing $>214,000$ spotted oligonucleotides, which represent almost every exon from coding mRNAs, microRNAs and long non-coding RNAs (scheme in Fig. 5A). This was first done in spinal cord tissue from animals at the preterminal stage of 14 months when maximal expression dysregulations are expected but may already be confounded by altered cellular composition. Filtering the data for significant effects at strong at least 2 -fold change identified 75 coding or non-coding transcripts. Significant subtle 1.2-fold changes of expression were documented for 6239 transcripts (Fig. 5B). This profiling approach was done again at the age of 10 weeks (Fig. 5C) when initial deficits of weight and spontaneous motor activity become apparent (Sen et al., 2019b), to filter those factors with consistent significance and dysregulation in the same direction at both ages, and to safeguard against expression changes due to altered cellular composition of the tissue at late ages. This expression survey at incipient pathology stage reproduced many significant effects, although with lower fold-changes. Only the Etnppl transcript was dysregulated more than 2-fold already at 10 weeks. Prominent (fold-change beyond 2.4-fold at 14 months) coding transcripts fulfilling all selection criteria are shown in Table 1.

In the subsequent bioinformatic evaluation we assessed all 1.2-fold expression dysregulations with nominal significance, if they were components of a pathway that showed enrichment after correction for multiple testing. The reasoning behind the screening of so subtle changes comes from research into Parkinson's disease (PD), where 2fold dosage increase of the disease protein alpha-synuclein was shown to trigger disease onset at ages of 30 years, 1.5-fold increase causes onset around 50 years, and 1.3-fold increase starts disease after 70 years of age (Book et al., 2018; Chiba-Falek and Nussbaum, 2001; Singleton et al., 2003). The survey of 1.2 -fold global transcriptome dysregulations in a PD mouse model correctly discovered neuroinflammatory changes as initial molecular pathology, which were later found to be crucial for a successful rescue (Sliter et al., 2018; Torres-Odio et al., 2017). Given that ATXN2 mutations also trigger mitochondrial dysfunction as in PD, and that SCA2 may manifest with a Parkinsonian phenotype (Park et al., 2015; Schols et al., 2015; Sen et al., 2016; Sen et al., 2017; Wang et al., 2015), we assessed here if distortion of such pathways becomes relevant by subtle dysregulations at several points.

At incipient disease stage of the Atxn2-CAG100-KIN mouse, a 1.2fold expression change was observed for 1,699 downregulations versus 2,887 upregulations; at preterminal age, the number of 1,514 downregulations appeared quite similar, but an increased number of 4,725 upregulations reflected the disease progression. The individual factors in their interaction clusters were visualized in STRING diagrams (Suppl. Fig. S5). Initially the upregulations were prominent (Suppl. Fig. S5A) for the ATXN2 interactor Pabpc1 mRNA, together with various other translation initiation and spliceosomal factors, in parallel to upregulations of acetylation and mitochondrial factors. This was accompanied by initial downregulations (Fig. S5B) for receptor tyrosine kinases that signal via the ATXN2-interactors SRC and GRB2, together with depletions for their downstream effectors, various MAP kinases and CAM kinases. Downregulations included pathway enrichments for potassium channels, synapse/axon factors, and cholesterol biosynthesis enzymes (statistics in Suppl. Table S1).

To reduce complexity at preterminal stage, only 2-fold expression changes were evaluated by STRING. The strong upregulations included immune defense and lysosome components, beyond the RNA-binding and bioenergetics proteins (Suppl. Fig. S5C). The strong downregulations again reflected deficits of potassium channels, synapse/axon factors, and cholesterol biosynthesis enzymes (Suppl. Fig. S5D; statistics in Suppl. Table S2).

\subsection{Pathway enrichments in the Transcriptome Analysis Console relate to ATXN2 interactome}

Bioinformatics via the Affymetrix Clariom Transcriptome Analysis Console (TAC) recognized enrichments for apparently diverse pathways at incipient stage (Suppl. Fig. S6) and preterminal stage of disease (Suppl. Fig. S7). As common denominator, all these pathways reflect 

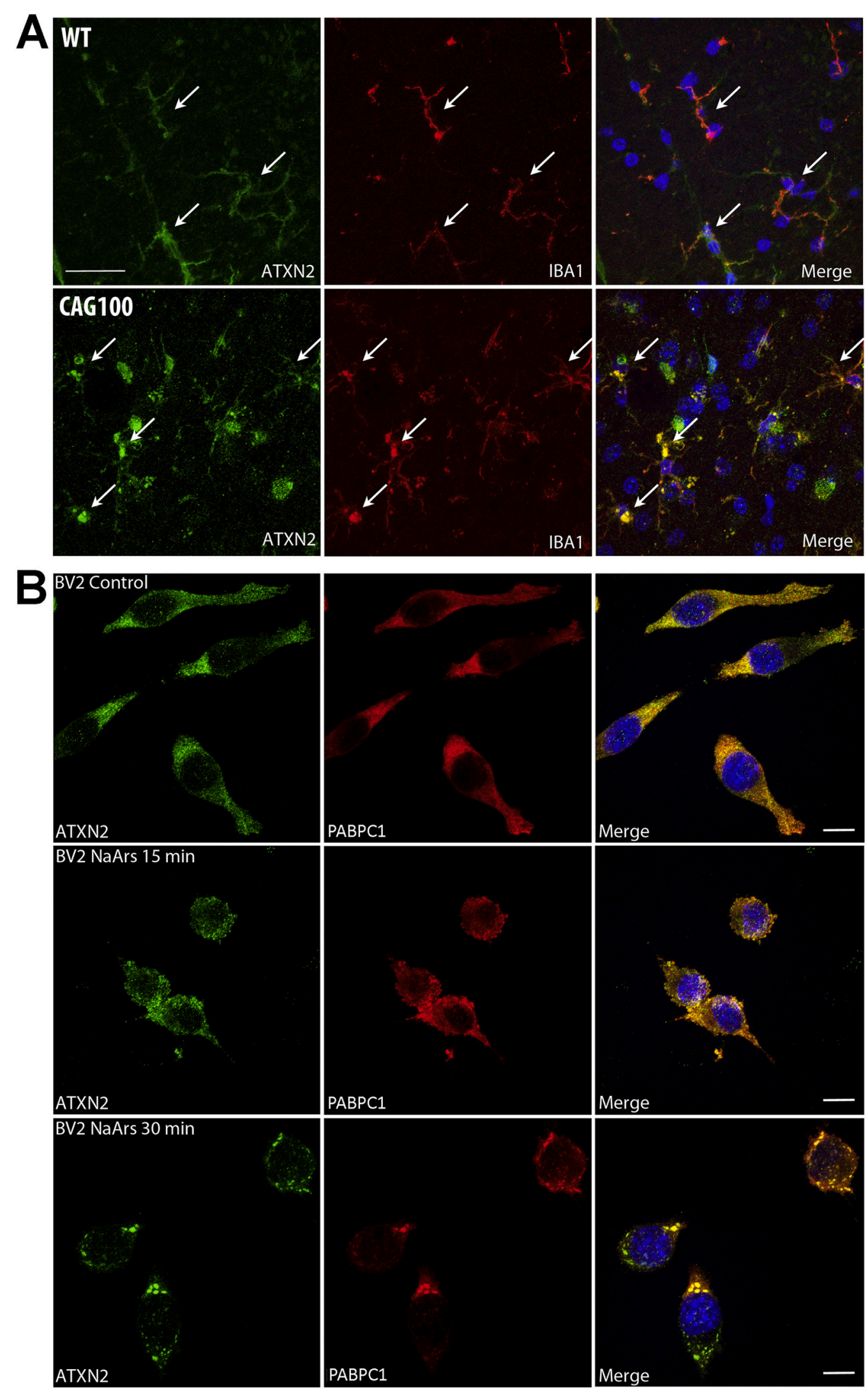

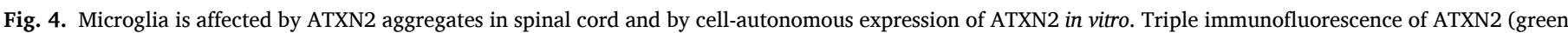

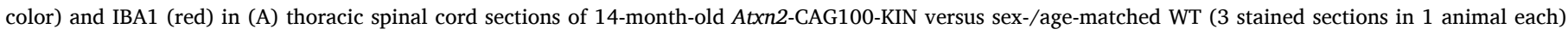

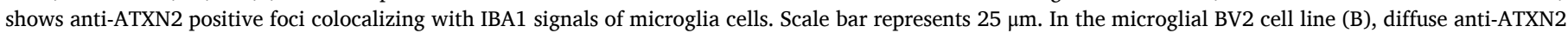

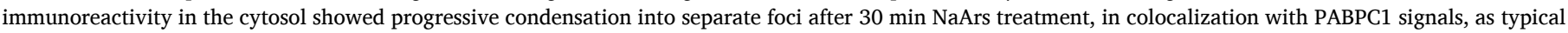
stress granule features. In the merged panels, nuclei are visualized stained with DAPI (blue). Scale bar represents $50 \mu \mathrm{m}$. 
Table 1

List of coding transcripts with dysregulation effect doubling over KIN lifespan. Candidate progression markers were selected from global transcriptome profiles upon $\geq 2$.4-fold change at 14 months of age, preceded by also significant $>1$.2-fold expression change at 10 weeks of age, in Atxn2-CAG100-KIN cervicothoracic spinal cord tissue. All such factors were downregulated, as illustrated by negative fold change effects. Significances shown were calculated by microarray Clariom D Transcriptome Analysis Console. Pathway component clusters are highlighted by colors, using red for cholesterol biosynthesis, purple for lipid metabolism, light green for axon, dark green for presynapse, gold for members of the ATXN2 interactome, rose for factors responsible for neurodegenerative disorders, beige for markers of distinct neuron populations. Authors' comments on pathway functions of disease implications are provided at the right margin.

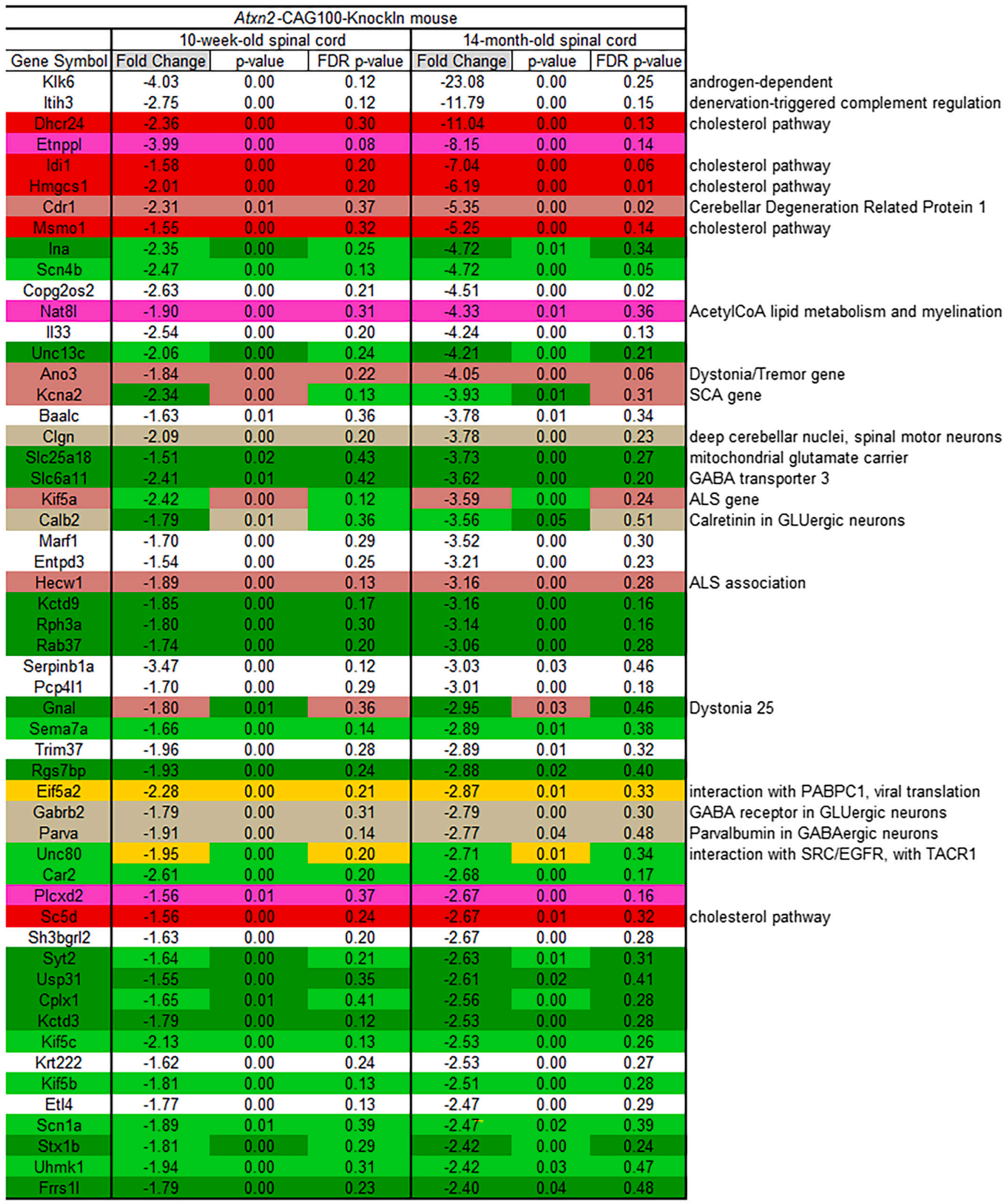


et al., 2019a). Interestingly, in the CAG100-KIN spinal cord expression profile (Suppl. Fig. S8C), significance was just achieved for the downregulation of Ttbk2, as a factor with causal role in tauopathies and TDP43 phosphorylation (Taylor et al., 2018).

3.8. Strongest progression of dysregulation for cholesterol biosynthesis and synapse/axon factors

Next, we attempted to identify molecular biomarkers of disease

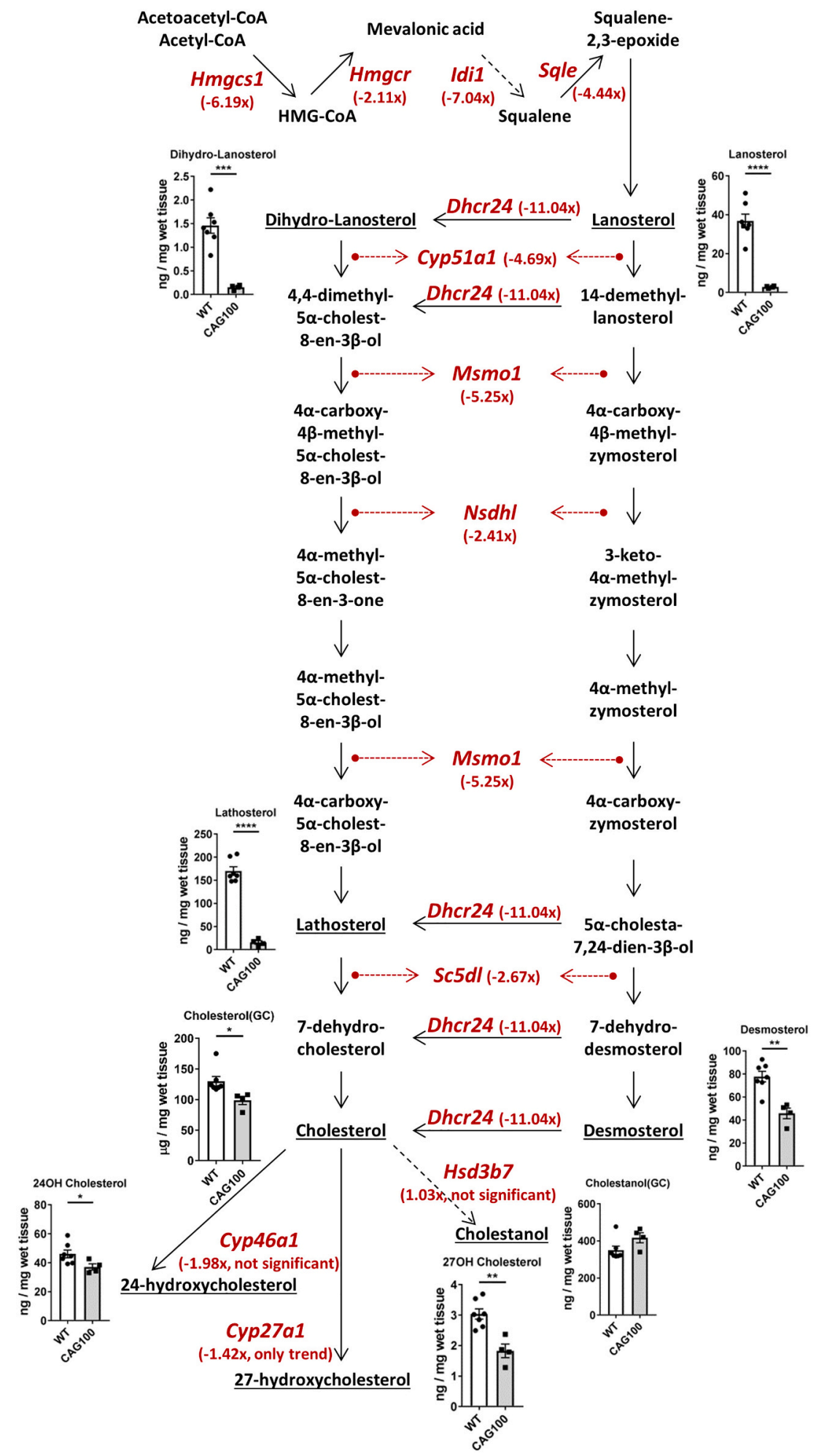

progression by selecting the $\geq 2$.4-fold dysregulations in the 14 -monthold spinal cord, which were also significant with at least $\geq 1$.2-fold expression changes in 10-week-old tissue. Table 1 shows the 51 factors that were defined by this approach. They were studied in great detail with respect to pathway enrichment, partially validated at RT-qPCR and immunoblot level (Suppl. Fig. S3 and S9 and Suppl. Table S5), and assessed regarding enzyme metabolic effects (Fig. 6). We prioritized them in view of the high importance of progression markers for the understanding of pathogenesis and the evaluation of neuroprotective

Fig. 6. Cholesterol biosynthesis pathways show suppressed expression of enzymes and deficient metabolism intermediates in KIN spinal cord. In this schematic presentation of cholesterol biosynthesis via lathosterol and via desmosterol, significant transcriptional downregulations of various enzymes are highlighted in red letters for each gene symbol and each foldchange. The quantification of several intermediate metabolites is illustrated by bar graphs. The gas chromatography analysis was performed in 12-month-old spinal cord from 7 WT versus 4 KIN mice, see Suppl. Table S5. 
therapies.

Table 1 uses red color to highlight the novel and crucial observation that factors of cholesterol metabolism are clustering among the strongest repression effects. This was evident upon automated STRING bioinformatics (Suppl. Fig. S5E; Suppl. Table S3). In addition, the androgen(a cholesterol-derived hormone) responsive Klk6 expression was downregulated (Yousef et al., 1999). Similarly, the Etnppl mRNA was decreased, and its activity is triggered by corticosteroids, which are derived from cholesterol (Fleshood and Pitot, 1970). It is noteworthy that the mRNA encoding the enzyme Nat8l is downregulated as well, reflecting the insidious depletion of the abundant brain metabolite $\mathrm{N}$ acetyl-aspartate (generated in neuronal mitochondria to shuttle metabolic substrates for oligodendroglia myelination), as an established imaging biomarker of advanced disease in SCA2 patients and in our mouse model (Sen et al., 2019b). Together, these findings suggest progressive and prominent deficits in acetyl-CoA supply, membrane phospholipid metabolism, myelin lipid synthesis, cholesterol biogenesis and hormone homeostasis.

\subsection{Quantification of cholesterol biogenesis pathway intermediate metabolites confirms significant deficits that cannot be compensated}

We wondered whether the cholesterol pathway dysregulation is a primary event that contributes to neurodegeneration or whether it is secondary, a consequence of membrane breakdown. The transcriptional downregulations of various enzymes in cholesterol biogenesis and turnover were confirmed by RT-qPCR (Suppl. Fig. S9). Are the mRNA levels of these enzymes repressed, because excessive amounts of free cholesterol are available in the aftermath of synapse and axon loss? Or is this transcript deficit responsible for cholesterol depletion? To assess this question, the intermediary metabolites of cholesterol metabolism were quantified by gas chromatography- mass spectrometry using selective ion detection methodology in spinal cord tissue at the age of 14 months. To distinguish the endogenous biosynthesis effects from the impact of dietary cholesterol via blood content in the tissues studied, the plant sterols campesterol and sitosterol were also quantified, thus demonstrating the contamination from serum to be minimal. Conversely to the significant increase of cholesterol in Atxn2-KO mouse blood (Lastres-Becker et al., 2008), a significant 0.76-fold decrease of cholesterol was documented (Suppl. Table S4). Also its degradation products $24 \mathrm{OH}$-cholesterol and $27 \mathrm{OH}$-cholesterol showed significant 0.80 -fold and 0.60 -fold deficits, respectively (Suppl. Table S4). Other known deficits of cholesterol biogenesis such as the Smith-Lemli-Opitz syndrome or desmosterolosis have a phenotype that includes mental retardation due to brain atrophy, with the membrane cholesterol deficit being partially compensated by the integration of precursor metabolites (Lee et al., 2013; Porter and Herman, 2011; Schaaf et al., 2011; Wechsler et al., 2003). Cholesterol biogenesis is also impaired in Huntington's disease, a neurodegenerative disorder again with autosomal dominant inheritance due to a polyQ expansion (Di Pardo et al., 2020). In autosomal recessive Spinocerebellar ataxia type 12 (SCAR12), mutations of WWOX trigger deficient steroidogenesis (Abdeen et al., 2013; Mallaret et al., 2014). In contrast, in our SCA2 mouse model the precursor metabolites showed massive deficits at multiple steps in both the Bloch pathway and the Kandutsch-Russell pathway, with 0.08-fold reduction for lanosterol at the beginning of post-squalene synthesis, and 0.09-fold reduction for lathosterol towards the end of the biosynthesis cascade (Fig. 6). Comparisons between the cholesterol enzyme expression deficits listed Table 1 and these metabolite quantifications demonstrate an excellent correlation of cholesterol precursor metabolite deficits (Fig. 6). Furthermore, the significant gradual downregulations of the biosynthesis enzymes Sqle, Sc5d, Nat8l, Etnppl and Plcxd2 indicate that cholesterol synthesis is affected already in the pre-squalene pathway and that the basal homeostasis of Acetyl-CoA and membrane phospho-lipids is also altered. These findings are in good agreement with global proteome profiles of Atxn2-null organisms, which demonstrated a prominent alteration of the breakdown of fatty acids and amino acids to Acetyl-CoA within mitochondria of mice, and demonstrated a prominent affection of the citric acid cycle in yeast (Meierhofer et al., 2016; Seidel et al., 2017a), jointly emphasizing a metabolic role for ATXN2. Altogether, expression profiles at initial and late disease stages are corroborated by lipid quantification studies, pointing to significant deficiencies of cholesterol and its precursors in the nervous tissue as a primary event of pathogenesis, which occurs so early and progresses so strongly that this pathway can serve as a sensitive and specific read-out in therapeutic trials.

\section{Discussion}

We have previously generated the novel Atxn2-CAG100-KnockIn mouse and shown for the cerebellum that (i) the temporal evolution of locomotor deficits and progressive atrophy, (ii) the spatial distribution of its pathology, and (iii) the neurochemical anomalies upon brain imaging faithfully reflect the known features of SCA2 (Sen et al., 2019b). For the spinal cord pathology as well, the current study confirms that this mouse mutant represents an authentic SCA2 model: Neurophysiologically, the distinctive features of SCA2 patients around clinical manifestation include an early sensory neuropathy by predominantly axonal lesion with signs of myelin damage (Bezerra et al., 2016; Pelosi et al., 2019; Rub et al., 2003a; Rub et al., 2003b; Rub et al., 2007; Velazquez-Perez et al., 2001; Velazquez-Perez et al., 2014), before the degeneration of lower and upper motor neurons starts with craniocervical preference (Velazquez-Perez et al., 2016a; Velazquez-Perez et al., 2016b; Velazquez-Perez et al., 2018; Velazquez-Perez et al., 2017a; Velazquez-Perez et al., 2017b). Similarly, the Atxn2-CAG100KIN showed sensory neuropathy as earliest peripheral manifestation of disease at the age of 9-10 months. As a pathological hallmark of spinal motor neuron affection, the progressive aggregation of TDP43, ATXN2 and other stress granule proteins was observed in the nervous tissue of human SCA2 (Elden et al., 2010; Koyano et al., 2014; Rub et al., 2013; Seidel et al., 2017b), and is also documented in this mouse model. In view of the rarity of SCA2 autopsies, no human expression profiles became available until now that could elucidate the pathological mechanisms in the affected tissue. Thus, the Atxn2-CAG100-KIN mouse provided a unique opportunity to explore in unbiased manner the impact of spinal pathology in SCA2 on RNA levels, and the molecular insights documented here are completely novel.

Overall, the pathway enrichments demonstrated a decreased expression for synaptic and axonal factors as well as cholesterol enzymes. This loss occurs in parallel to astrogliosis, as expected, but interestingly, our data represent also the first report that enhanced expression of lysosomal factors and microgliosis are prominent at late disease stages. Mechanistically, the activated microglia may be partially due to the presence of ATXN2 aggregates, which are presumably extruded from neurons and internalized by phagocytosis, but this may also be exacerbated by the robust expression of expanded ATXN2 in stressed microglia cells, as a cell-autonomous trigger.

The comparison of significant 2.4-fold dysregulations at preterminal stage versus significant 1.2 -fold changes at incipient pathology in Table 1 identified a total of 54 genes with progressively changed expression during the disease course, including conspicuously strong downregulations for several enzymes of cholesterol biogenesis, which were validated by RT-qPCR and metabolite quantification. Another recent report of spinal cord transcriptome data from a mouse with overexpression of the entire polyQ-expanded Ataxin-2 gene within a bacterial artificial chromosome also documented differentially expressed genes within the known ALS pathomechanism and the cholesterol pathway, together with fatty acid and immunity alterations as the prominent effects, but did not assess individual metabolites (Scoles et al., 2020). A recent analysis of mice with constitutive KnockOut of the sequence homologous Ataxin-2-like (Atxn2l) demonstrated mid-gestational lethality and cellular phenotypes known from 
cholesterol deficit states, together with an influence of cholesterol supplementation on the Atxn2l-promoter activity (Key et al., 2020). It is relevant to note that also in a mouse model of SCA3 (Machado-Joseph disease), recent global transcriptome profiling demonstrated a significant enrichment of cholesterol biogenesis dysregulations in brainstem, but increased levels of ceramides, di- and triglycerides in blood (Toonen et al., 2018). Furthermore, for SCA3 it was shown that restoration of brain cholesterol homeostasis has therapeutic potential (Nobrega et al., 2019). Our observation of reduced cholesterol biogenesis in the Atxn2CAG100-KIN mouse is easily related with the documented depletion of peripheral fat tissues in SCA2 (Medrano-Montero et al., 2018), and with findings of early demyelination in this CAG100-KIN mouse and in SCA2 patients (Gierga et al., 2005; Paciorkowski et al., 2011; Rub et al., 2003b). The quantitative analysis of several intermediate steps of cholesterol metabolism in the KIN spinal cord confirmed a significant deficit of cholesterol and massive deficiencies for several precursors. Even stronger depletion of cholesterol, as well as very-long-chain sphingomyelins (products of the ataxia disease gene Elovl4, also downregulated in the CAG100-KIN, see Suppl. Fig. S7C), was recently documented in a SCA2 patient cerebellum (Sen et al., 2019a). Cholesterol is a requirement for the synthesis of sex hormones and of corticosteroid stress hormones, so this might explain also the gradually declining expression of androgen-dependent factor Klk6 as the most dramatic downregulation effect. The progressive loss of $C d r 1$ expression might be explained in a similar context, since it is known to be regulated by the myelination factor Prnp (prion protein). Our study is not the first report of a connection between RNA-binding proteins such as ATXN2, TDP43 or TIA1 on the one hand and obesity or cholesterol on the other hand. Transgenic overexpression or KnockIn of TDP43 in mouse triggered weight loss and increased fat deposition with elevated HDL cholesterol in blood (Stallings et al., 2013; Stribl et al., 2014); LXRbeta ${ }^{-/-}$mice showed TDP43 aggregation together with higher brain cholesterol (Kim et al., 2008); conversely, the KnockOut of TDP43 results in dramatic loss of body fat (Chiang et al., 2010); similarly, the KnockOut of TIA1 triggers upregulation of fat storage factors (Heck et al., 2014) and the KnockOut of ATXN2 triggers hypercholesterolemia (Lastres-Becker et al., 2008). In ALS, the spinal cord ventral horn of patients showed a significant decrease of cholesterol (Hanrieder and Ewing, 2014), whereas in blood an increase of cholesterol was documented (Gonzalez De Aguilar, 2019). Controversial reports exist whether the use of cholesterol-lowering medication such as statins enhances or reduces ALS risk (Freedman et al., 2018; Golomb et al., 2009). In SCA2, the lipid metabolism seems to be dysregulated at a more basic level than cholesterol generation, given that expression of $\mathrm{Nat8l}$ as the enzyme responsible for $\mathrm{N}$-acetylaspartate (NAA) synthesis was gradually declining in Atxn2-CAG100-KIN spinal cord. Indeed, spinal NAA decreases during the disease course of ALS patients (Carew et al., 2011; Ikeda et al., 2013; Sangaraju et al., 2017).

Overall, the gradual expression reduction of several cholesterol enzymes is prominent and strong, so it may become useful for the future validation of progression biomarkers in SCA2 patient samples. However, cholesterol depletion is hardly a SCA2-specific feature. At present, clinical trials in SCA2 depend on the quantification of clinical, neurophysiological and imaging features with documented progression, such as the clinical SARA score (Diallo et al., 2018; Jacobi et al., 2015), quantification of sensory neuropathy (Velazquez-Perez et al., 2010), saccade slowing (Rodriguez-Labrada et al., 2016; Velazquez-Perez et al., 2009; Velazquez-Perez et al., 2004), periodic leg movements during sleep (Rodriguez-Labrada et al., 2019; Velazquez-Perez et al., 2011) and brain volumetry (Adanyeguh et al., 2018; Reetz et al., 2018). Since any improvement in these disease features occurs over extended periods of time, there is an unmet need to characterize molecular biomarkers that mirror therapeutic benefits very rapidly. Among such markers, it is important to distinguish secondary from primary events, to differentiate events that affect any cell type from those that characterize the most vulnerable cell population, and to integrate their contribution into plausible scenarios, a speculative effort that is undertaken in the subsequent paragraphs.

Among the strong effects in Table 1, any neuron population and glia cell type also would be affected by the depletion of Eif5a2 transcripts encoding a translation initiation factor. While the cholesterol depletion might be a secondary event, the progressive expression reduction of Unc80 and Eif5a2 concerns factors within the ATXN2 interactome and might thus represent primary and specific effects of SCA2 pathology. ATXN2 is associated to PABPC1 in the ribosomal translation complex (Damrath et al., 2012; Fittschen et al., 2015; Lastres-Becker et al., 2016; van de Loo et al., 2009), but its exact role there was never understood. The yeast ortholog of EIF5A is known for its role in cell wall integrity. The EIF5A protein is the only factor containing the unusual conserved amino acid hypusine, was reported to aid translation of polyprolinemotifs, modulates co-translational ER translocation, promotes stress granule assembly and mRNA decapping, is key for pancreatic beta-cell inflammation in diabetes mellitus, and mediates the effect of polyamines on neuronal process extension as well as survival via control of autophagy-controlling factors TFEB and ATG3. EIF5A also regulates mitochondrial respiration via initiation from alternative start codons. In addition, EIF5A restricts RNA virus infections (Caceres et al., 2016; Ganapathi et al., 2019; Gutierrez et al., 2013; Huang et al., 2007; Li et al., 2010; Lubas et al., 2018; Maier et al., 2010; Mandal et al., 2016; Mounce et al., 2017; Pereira et al., 2016; Puleston et al., 2019; Rossi et al., 2014; Zhang et al., 2019). It is important to note in this context that ATXN2 is cleaved by Coxsackie-virus and Polio-virus, during their optimized efforts to diminish host cell defenses against invading viral RNA (Jagdeo et al., 2018). Therefore, we used RT-qPCR to systematically assess expression dysregulation triggered by ATXN2 mutations in cerebellum as an independent validation effort, examining various factors that are involved in the RNA translation and shuttling, versus degradation of toxic viral RNA (Suppl. Table S5). Indeed, this approach confirmed that independent from polyQ expansions, altered ATXN2 functions lead to significant expression downregulations for Eif5a2. A similar downregulation was observed for the RNA decapping enzyme $D c p 2$ that co-localizes with the PABPC1 interactor TOB1 in P-bodies, but unlike ATXN2 and PABPC1 is not sequestrated to viral production factories around lipid droplets (Ariumi et al., 2011; Shapouri et al., 2016). The concept of excessive exposure to toxic RNA was further substantiated, when RT-qPCR experiments validated microarray data in 14month-old Atxn2-CAG100-KIN spinal cord on the 1.6-fold transcriptional upregulation of Rnaset2 (Suppl. Fig. S9). This ribonuclease with localization in lysosomes is responsible for the degradation of mitochondrial RNA and mitochondria-associated ribosomal RNA (Haud et al., 2011; Huang et al., 2018). Mutations in Rnaset2 cause neuroinflammation in a syndrome with cystic leukoencephalopathy (Henneke et al., 2009). The age-progressive release of toxic RNA and DNA from dysfunctional mitochondria was recently shown to constitute a key stress for innate immune defenses and for the neuroinflammation underlying Parkinsonian brain atrophy (Dhir et al., 2018; Sliter et al., 2018; West and Shadel, 2017).

Another factor in Table 1 with functions at a physiological site of ATXN2 localization and a steady expression downregulation during the neurodegenerative process was UNC80, which is activated by the tachykinin receptor 1 (TACR1) via SRC as an interactor of ATXN2 (Drost et al., 2013; Lu et al., 2009; Nonis et al., 2008). TACR1 binds substance-P selectively for the sensory perception of itch, pain and inflammation. It is abundant in the spinal cord dorsal horn neurons but is also a component of microglia sensing (Carniglia et al., 2017; Zieglgansberger, 2019). Tachykinin levels in the cerebrospinal fluid of ALS patients have been found elevated (Matsuishi et al., 1999). Furthermore, TACR1 accumulation was documented in the SOD1 mouse model of ALS and its pharmacological inhibition was found to be neuroprotective (Caioli et al., 2011; Li et al., 2015; Sirianni et al., 2015). In the Atxn2-CAG100KIN spinal cord, a significant decrease of Tacr1 mRNA levels was evident already at the exceptionally early age of 3 months, and remained 
similarly strong until the age of 14 months (Suppl. Fig. S9). This observation provides a possible molecular correlate for our phenotypic finding that sensory pathology precedes motor pathology in SCA2. Furthermore, the strongest upregulation among all transcripts ( $\sim 45$-fold upon RT-qPCR, Suppl. Fig. S9) concerned the Glycoprotein NMB (aka osteoactivin or Hematopoietic Growth Factor Inducible Neurokinin-1 = HGFIN), encoded by Gpnmb. This neural-expressed glycoprotein interacts with substance-P, it activates SRC signaling, its depletion reduces neuropathic pain, and it can be induced by lysosomal stress also in microglia (Gabriel et al., 2014; Hou et al., 2015; Maric et al., 2015; Rameshwar, 2012). It is highly relevant to note that GPNMB has a neuroprotective role for TDP43 toxicity or ALS (Nagahara et al., 2017; Tanaka et al., 2012). GPNMB was also described as neurodegeneration biomarker in Alzheimer's, Parkinson's, Gaucher's disease and PLOSL leukodystrophy (Huttenrauch et al., 2018; Iwaki et al., 2019; Moloney et al., 2018; Murugesan et al., 2018; Satoh et al., 2019).

Among the strong dysregulations in Table 1, progressively reduced expression for several known neurodegeneration genes provides novel insights into the spinal cord atrophy process in this SCA2 model,: firstly, Cdr1 (encoding Cerebellar Degeneration Related Protein 1, aka CDR62 or CDR34 or Yo-antigen) downregulation relates to its well-known autoimmune depletion as a cause of paraneoplastic ataxia (Bolla and Palmer, 1997) - it is interesting to note that Cdr1 expression is induced by the myelination factor Prion protein (Satoh and Yamamura, 2004); secondly, the Spinocerebellar Ataxia gene and voltage-gated potassium channel Kcna2, which is preferentially expressed in afferent synapses onto the degenerating neurons (Helbig et al., 2016; Xie et al., 2010); it is interesting to note that the parallel reduction of Kctd3 and Kctd9 affects two factors with potassium channel tetramerization domains; thirdly, the ALS disease gene Kif5a (Brenner et al., 2018; Nicolas et al., 2018) and its interactors Kif5b and Kif5c, which encode factors of axonal transport; Kif5a clusters with the progressive dysregulations of Uhmk1 (aka Ser/Thr-Protein kinase KIS) and Ina (aka internexin neuronal intermediate filament) in Table 1 , since these factors relate to ribonucleoprotein and stress granule transport (Cambray et al., 2009; Furukawa et al., 2015; Liu and Szaro, 2011). In the same context, the progressive expression downregulation of Hecw1 seems relevant, since this ubiquitination enzyme is responsible for the degradation of the ALS disease protein SOD1, is sequestrated into the cytosolic aggregates in ALS neurons, and its mutation leads to ALS-like phenotypes in mouse (Miyazaki et al., 2004; Zhang et al., 2011); fourth, the downregulation of Ano3 is important in view of its impact on tremor and dystonia (Charlesworth et al., 2012; Stamelou et al., 2014); similarly, the reduced expression of Gnal encoding the G-protein G(olf) alpha, and of Rgs7bp encoding R7bp as general regulator of G-protein signaling appears relevant, in view of Gnal mutations triggering dystonia type 25 and the key role of R7bp in spinal afferents (Fuchs et al., 2013; Liapis et al., 2012; Pandey et al., 2017); fifth, the insidious reduction of $S c n 4 b$ mRNA seems relevant, given that $S c n 4 b$-null mice show motor coordination and balance deficits (Ransdell et al., 2017), that Scn $4 b$ expression depends on GABA-A signaling (Ponomarev et al., 2006) and that Scn $4 b$ depletion was also observed in the striatum affected by polyglutamineneurotoxicity due to Huntington's disease mutation (Oyama et al., 2006).

Evaluating the $>2$.4-fold changes in Table 1 further, several calciumbinding factors with selective expression in different neuron populations seemed relevant and were highlighted in beige color: The progressive decrease of Calb2 mRNA encoding Calretinin in cerebellum mirrors specifically the GLUergic parallel fibers, whereas the depletion of Parvalbumin (encoded by the Parva gene) is a marker of GABAergic Purkinje neurons. Both are lost from ALS spinal cord tissue (Canet-Pons et al., 2018; Hayashi et al., 2013; Maskey et al., 2010; Wishart et al., 2012). The parallel reduction of Clgn mRNA encoding the endoplasmic reticulum chaperone Calmegin represents cerebellar deep neurons and spinal motor neurons, according to the Allen mouse spinal cord in-situ hybridization data. These observations suggest that the degeneration affects several neural projections in parallel rather than in a hierarchical time-course.

The strongest progression marker in Table 1 was the decreased expression of the Klk6 gene, which contains a purine repeat similar to the Friedreich ataxia gene and shows dysregulated expression during the neurodegenerative process of Alzheimer's disease (Singh and Rajeswari, 2015). Similarly, the dysregulation of Ina expression and of Il33 in Table 1 for the CAG100-KIN were similarly reported for ALS (Hawley et al., 2019; Lin et al., 2012). The progressive decrease of Unc13c mRNA levels in Table 1 reflects the specific motor deficits of these mice, in view of its key role in cerebellar parallel fibers for fast reflexes and motor learning (Augustin et al., 2001; Netrakanti et al., 2015); the depletion of Slc6a11 encoding the GABA-transporter-3, and of Gabrb2 encoding the GABA-A-receptor-beta-2, in CAG100-KIN clearly mirrors the dysfunction of GABA-ergic signaling in the spinal dorsal horn and in cerebellar Purkinje cells (Guo et al., 2012; Kataoka et al., 2013); the deficiency of Itih3-encoded protein complexes was documented in Table 1 and occurs similarly after denervation, leading to diminished exploration and anxiety-like behavior in mice (Businaro et al., 2001; Goulding et al., 2019; Okroj et al., 2012).

From the expression profiles in the KIN spinal cord, two molecular cascades can be assembled as plausible scenarios, both culminating in microgliosis. Firstly, as a putative correlate of the sensory neuropathy the transcript expression of several key factors in afferent signaling changed. The downregulation of the itch/pain-related Substance-P receptor Tacr1 reached significance particularly early at the age of 3 months. The Tacr1-dependent neuronal excitability factor Unc80, which encodes a scaffold for the ATXN2-interactor SRC, was identified as one of the best biomarkers for disease progression. The afferent excitability problem seems to involve altered potassium homeostasis, in view of the conspicuous expression dysregulation of several presynaptic $\mathrm{K}^{+}$channels (Ataxia disease genes Kcnj10, Kcnc3, Kcna1 in Suppl. Fig. S7C; Kcna2 with $\mathrm{K}^{+}$-channel tetramerization factors Kctd9 and Kctd3 in Table 1). This observation is in good agreement with a previous report that impaired excitability of Purkinje neurons in a SCA2 mouse model was rescued by modulation of $\mathrm{K}^{+}$dependent hyperpolarization (Egorova et al., 2016). Also the expression dysregulation of Ano3 and Gnal, two disease genes responsible for dystonia and tremor, may be caused within the same sensory pathway. In this context, it is important to consider that SCA2 mutations always trigger intention tremor and may have a clinical presentation mainly with tremor or dystonia (Cheng et al., 2018; Freund et al., 2007; Kuo et al., 2017; Markovic et al., 2016). At late stage, the strongest upregulation among all transcripts concerned the Substance-P interactor Gpnmb, which can be strongly induced in microglia cells by lysosomal stress and exerts a neuroprotective role in TDP43 pathology.

Secondly, a molecular cascade was documented as correlate of motor neuron affection by RNA toxicity, in excellent overlap with the pathogenesis of ALS. The downregulation of Eif5a2 may be a primary consequence of ATXN2 expansion, given that both factors colocalize in the ribosomal translation complex and that both act in the defense against viral RNAs. Also the accumulation of the toxic DNA/RNA sensor and stress granule assembly factor PQBP1 (Kunde et al., 2011) may be a direct consequence of its sequestration by polyQ-expanded ATXN2. The downregulation of $D c p 2$ as RNA degrading enzyme in the P-body may reflect the pathological retention of RNAs in stress granules affected by ATXN2 aggregation. In contrast, the upregulation of Rnaset2 is probably a compensatory effort, since this enzyme has the ability to degrade inappropriately methylated RNAs, which are released from dysfunctional mitochondria in ever higher quantities during the ageing process. Altogether, the accumulation of toxic RNAs seems to be reflected by the transcriptional induction of sensors such as Trl7, of the signaling factor Ripk1, the particularly early elevation of lysosomal PGRN protein and the increase of cytosolic CASP3 protein levels, which promote the aggregation of TDP43 at stress granules. A known correlate of this cascade in SCA2 pathology is probably the accumulation of Staufen 1 as a marker 
of neuronal RNA granules, which is also recruited into ATXN2 aggregates (Paul et al., 2018). Given that KIF5 is crucial for anterograde transport of virus particles (DuRaine et al., 2018), the progressive expression downregulation of all three KIF5 subunits in the KIN spinal cord might constitute a protective antiviral response within neurons. The progressive downregulation of Hecw1 expression is known to modulate the vulnerability of motor neurons by reduced degradation of misfolded SOD1, which was shown to affect stress granule dynamics via interaction with G3BP1 (Gal et al., 2016). It is important to note that the permanent cytosolic sequestration of such nucleotide processing factors by ATXN2 aggregates would lead not only to impaired RNA quality control, but also to altered defenses of motor neurons e.g. against poliovirus RNA infections (Dougherty et al., 2015; Marin et al., 2008; Thiagarajan et al., 2011; White and Lloyd, 2011). Of course, the hierarchical sequence of events in both scenarios may be more complex, but these dysregulations clearly stood out by fold-change and significance levels in the global transcriptome profiles of presymptomatic and preterminal KIN spinal cord (Table 1).

Overall, the novel observations that ATXN2 accumulation toxicity alters prominently microglial responses, RNA toxicity, the expression of Eif5a2, Unc80 and $K i f 5 a / b / c$, as well as cholesterol biosynthesis, conjointly point to an important role of ATXN2 for processes that modulate plasma membranes regarding composition, integrity, and internalization. However, this preliminary evidence has relevant limitations. Most importantly, the present exploration into global transcriptome levels is focused at ATXN2 effects on RNA stability, while the ATXN2 effects on global mRNA translation during periods of growth versus stress remain to be documented in future mass spectrometry projects, e.g. in organotypic slice cultures with documentation of the steady-state proteome versus the short-term translatome (Klann et al., 2020). In addition, the cell-autonomous pathology in diverse cell populations of the central nervous system should be assessed in depth via conditional mouse mutants, rather than the microglial cell line cultures employed here.

In conclusion, the spinal cord of our new Atxn2-CAG100-KIN mouse mutant revealed first insights into the molecular pathogenesis of SCA2. Sensory and motor affection involves prominently a loss of axonal and presynaptic factors, in parallel to depletion of cholesterol and phospholipid enzymes. Various molecular pathways are irritated by the sequestration of ATXN2 interactor molecules into aggregates, principally the role of stress granules in the defense against toxic RNAs. Several other altered pathways such as ribosomal translation, calcium homeostasis and cholesterol biogenesis occur at the endoplasmic reticulum, where ATXN2 was shown to play a crucial role for structure and dynamics according to studies in C. elegans and D. melanogaster (Del Castillo et al., 2019). SCA2 pathogenesis has considerable overlap with the mechanisms documented in other ataxias, dystonias, and with ALSassociated features such as RNA toxicity and endoplasmic reticulum dysfunction (Taylor et al., 2016).

Supplementary data to this article can be found online at https://doi. org/10.1016/j.nbd.2021.105289.

\section{Acknowledgements}

For their technical assistance, we are grateful to Birgitt MeseckSelchow and Gabriele Köpf in Frankfurt, to Jérome Sinniger in Strasbourg, to Michaela Neuenkirch in Langen, to Anja Kerksiek for technical assistance to perform sterol and oxysterol analysis, and to the staff of the ZFE animal facility at the Goethe University in Frankfurt. For financial support, we thank the Deutsche Forschungsgemeinschaft (grants AU96/ 11-1 and 11-3 to GA). GA receives financial support from Roche Pharma and Takeda Pharma for further projects on ATXN2.

\section{References:}

Abdeen, S.K., et al., 2013. Conditional inactivation of the mouse Wwox tumor suppressor gene recapitulates the null phenotype. J. Cell. Physiol. 228, 1377-1382.

Adanyeguh, I.M., et al., 2018. Autosomal dominant cerebellar ataxias: imaging biomarkers with high effect sizes. NeuroImage. Clin. 19, 858-867.

Almaguer-Mederos, L.E., et al., 2010. Estimation of the age at onset in spinocerebellar ataxia type 2 Cuban patients by survival analysis. Clin. Genet. 78, 169-174.

Al-Ramahi, I., et al., 2007. dAtaxin-2 mediates expanded Ataxin-1-induced neurodegeneration in a Drosophila model of SCA1. PLoS Genet. 3, e234.

Ariumi, Y., et al., 2011. Hepatitis C virus hijacks P-body and stress granule components around lipid droplets. J. Virol. 85, 6882-6892.

Auburger, G., et al., 1990. Autosomal dominant ataxia: genetic evidence for locus heterogeneity from a Cuban founder-effect population. Am. J. Hum. Genet. 46, 1163-1177.

Auburger, G., et al., 2014. 12q24 locus association with type 1 diabetes: SH2B3 or ATXN2? World J. Diabetes 5, 316-327.

Auburger, G., et al., 2017. Efficient prevention of neurodegenerative diseases by depletion of starvation response factor Ataxin-2. Trends Neurosci. 40, 507-516.

Auburger, G.W., 2012. Spinocerebellar ataxia type 2. Handb. Clin. Neurol. 103, 423-436.

Augustin, I., et al., 2001. The cerebellum-specific Munc13 isoform Munc13-3 regulates cerebellar synaptic transmission and motor learning in mice. J. Neurosci. 21, 10-17.

Banks, C.A., et al., 2016. TNIP2 is a hub protein in the NF-kappaB network with both protein and RNA mediated interactions. Mol. Cell. Proteomics 15, 3435-3449.

Bar, D.Z., et al., 2016. Cell size and fat content of dietary-restricted Caenorhabditis elegans are regulated by ATX-2, an mTOR repressor. Proc. Natl. Acad. Sci. U. S. A. 113, E4620-E4629.

Baumer, D., et al., 2014. FTLD-ALS of TDP-43 type and SCA2 in a family with a full ataxin-2 polyglutamine expansion. Acta Neuropathol. 128, 597-604.

Becker, L.A., et al., 2017. Therapeutic reduction of ataxin-2 extends lifespan and reduces pathology in TDP-43 mice. Nature. 544, 367-371.

Beel, S., et al., 2017. Progranulin functions as a cathepsin D chaperone to stimulate axonal outgrowth in vivo. Hum. Mol. Genet. 26, 2850-2863.

Belal, S., et al., 1994. Clinical and genetic analysis of a Tunisian family with autosomal dominant cerebellar ataxia type 1 linked to the SCA2 locus. Neurology. 44, 1423-1426.

Bernard-Marissal, N., et al., 2018. Endoplasmic reticulum and mitochondria in diseases of motor and sensory neurons: a broken relationship? Cell Death Dis. 9, 333.

Bezerra, M.L., et al., 2016. Pattern of Peripheral Nerve Involvement in Spinocerebellar Ataxia Type 2: a Neurophysiological Assessment. Cerebellum. 15, 767-773.

Blasi, E., et al., 1990. Immortalization of murine microglial cells by a v-raf/v-myc carrying retrovirus. J. Neuroimmunol. 27, 229-237.

Bolla, L., Palmer, R.M., 1997. Paraneoplastic cerebellar degeneration. Case report and literature review. Arch. Intern. Med. 157, 1258-1262.

Book, A., et al., 2018. A Meta-Analysis of alpha-Synuclein Multiplication in Familial Parkinsonism. Front. Neurol. 9, 1021.

Brenner, D., et al., 2018. Hot-spot KIF5A mutations cause familial ALS. Brain J. Neurol. 141, 688-697.

Businaro, R., et al., 2001. Altered balance of proteinase inhibitors in atrophic muscle after denervation. Ital. J. Anatomy Embryol. = Arch. ital. Anatomia Embriol. 106, $159-165$.

Caceres, C.J., et al., 2016. Targeting deoxyhypusine hydroxylase activity impairs capindependent translation initiation driven by the 5'untranslated region of the HIV-1, HTLV-1, and MMTV mRNAs. Antivir. Res. 134, 192-206.

Caioli, S., et al., 2011. Substance P receptor activation induces downregulation of the AMPA receptor functionality in cortical neurons from a genetic model of Amyotrophic Lateral Sclerosis. Neurobiol. Dis. 44, 92-101.

Cambray, S., et al., 2009. Protein kinase KIS localizes to RNA granules and enhances local translation. Mol. Cell. Biol. 29, 726-735.

Cameron, B., et al., 2012. Loss of interleukin receptor-associated kinase 4 signaling suppresses amyloid pathology and alters microglial phenotype in a mouse model of Alzheimer's disease. J. Neurosci. 32, 15112-15123.

Canet-Pons, J., et al., 2018. Ataxia telangiectasia alters the ApoB and reelin pathway. Neurogenetics. 19, 237-255.

Carew, J.D., et al., 2011. Presymptomatic spinal cord neurometabolic findings in SOD1positive people at risk for familial ALS. Neurology. 77, 1370-1375.

Carniglia, L., et al., 2017. Neuropeptides and Microglial Activation in Inflammation, Pain, and Neurodegenerative Diseases. Mediat. Inflamm. 2017, 5048616.

Casula, M., et al., 2011. Toll-like receptor signaling in amyotrophic lateral sclerosis spinal cord tissue. Neuroscience. 179, 233-243.

Charlesworth, G., et al., 2012. Mutations in ANO3 cause dominant craniocervical dystonia: ion channel implicated in pathogenesis. Am. J. Hum. Genet. 91, 1041-1050.

Cheng, N., et al., 2018. SCA2 presenting as a focal dystonia. J. Clin. Move. Disord. 5, 6.

Chen-Plotkin, A.S., et al., 2010. TAR DNA-binding protein 43 in neurodegenerative disease. Nat. Rev. Neurol. 6, 211-220.

Chiang, P.M., et al., 2010. Deletion of TDP-43 down-regulates Tbc1d1, a gene linked to obesity, and alters body fat metabolism. Proc. Natl. Acad. Sci. U. S. A. 107, 16320-16324.

Chiba-Falek, O., Nussbaum, R.L., 2001. Effect of allelic variation at the NACP-Rep1 repeat upstream of the alpha-synuclein gene (SNCA) on transcription in a cell culture luciferase reporter system. Hum. Mol. Genet. 10, 3101-3109.

Damrath, E., et al., 2012. ATXN2-CAG42 sequesters PABPC1 into insolubility and induces FBXW8 in cerebellum of old ataxic knock-in mice. PLoS Genet. 8, e1002920.

Del Castillo, U., et al., 2019. Conserved role for Ataxin-2 in mediating endoplasmic reticulum dynamics. Traffic. 20, 436-447. 
DeMille, D., et al., 2015. PAS kinase is activated by direct SNF1-dependent phosphorylation and mediates inhibition of TORC1 through the phosphorylation and activation of Pbp1. Mol. Biol. Cell 26, 569-582.

Dhir, A., et al., 2018. Mitochondrial double-stranded RNA triggers antiviral signalling in humans. Nature. 560, 238-242.

Di Pardo, A., et al., 2020. Mutant huntingtin interacts with the sterol regulatory element binding proteins and impairs their nuclear import. Hum. Mol. Genet. 29, 418-431.

Diallo, A., et al., 2018. Survival in patients with spinocerebellar ataxia types 1, 2, 3, and 6 (EUROSCA): a longitudinal cohort study. Lancet Neurol. 17, 327-334.

Diner, B.A., et al., 2015. The functional interactome of PYHIN immune regulators reveals IFIX is a sensor of viral DNA. Mol. Syst. Biol. 11, 787.

Dougherty, J.D., et al., 2015. Multiple poliovirus proteins repress cytoplasmic RNA granules. Viruses. 7, 6127-6140.

Drost, J., et al., 2013. Ataxin-2 modulates the levels of Grb2 and SRC but not ras signaling. J. Mol. Neurosci.: MN 51, 68-81.

DuRaine, G., et al., 2018. Kinesin-1 Proteins KIF5A, -5B, and -5C promote anterograde transport of herpes simplex virus enveloped virions in axons. J. Virol. 92 e01269-18.

Egorova, P.A., et al., 2016. In vivo analysis of cerebellar Purkinje cell activity in SCA2 transgenic mouse model. J. Neurophysiol. 115, 2840-2851.

Elden, A.C., et al., 2010. Ataxin-2 intermediate-length polyglutamine expansions are associated with increased risk for ALS. Nature. 466, 1069-1075.

Estrada, R., et al., 1999. Spinocerebellar ataxia 2 (SCA2): morphometric analyses in 11 autopsies. Acta Neuropathol. 97, 306-310.

Fittschen, M., et al., 2015. Genetic ablation of ataxin-2 increases several global translation factors in their transcript abundance but decreases translation rate. Neurogenetics. 16, 181-192.

Fitzgerald, K.D., Semler, B.L., 2013. Poliovirus infection induces the co-localization of cellular protein SRp20 with TIA-1, a cytoplasmic stress granule protein. Virus Res. 176, 223-231.

Fleshood, H.L., Pitot, H.C., 1970. The metabolism of O-phosphorylethanolamine in animal tissues. II. Metabolic regulation of O-phosphorylethanolamine phospho-lyase in vivo. Arch. Biochem. Biophys. 141, 423-429.

Freedman, D.M., et al., 2018. Relationship of statins and other cholesterol-lowering medications and risk of amyotrophic lateral sclerosis in the US elderly. Amyotrop. Lateral Sclerosis Frontotemp. Degener. 19, 538-546.

Freund, H.J., et al., 2007. Subthalamic-thalamic DBS in a case with spinocerebellar ataxia type 2 and severe tremor-A unusual clinical benefit. Move. Disord: Off. J. Move. Disord. Soc. 22, 732-735.

Fuchs, T., et al., 2013. Mutations in GNAL cause primary torsion dystonia. Nat. Genet. 45, 88-92.

Furukawa, M.T., et al., 2015. Interaction and colocalization of HERMES/RBPMS with NonO, PSF, and G3BP1 in neuronal cytoplasmic RNP granules in mouse retinal line cells. Genes Cells: Devot. Mol. Cell. Mech. 20, 257-266.

Gabriel, T.L., et al., 2014. Lysosomal stress in obese adipose tissue macrophages contributes to MITF-dependent Gpnmb induction. Diabetes. 63, 3310-3323.

Gal, J., et al., 2016. ALS mutant SOD1 interacts with G3BP1 and affects stress granule dynamics. Acta Neuropathol. 132, 563-576.

Ganapathi, M., et al., 2019. Recessive Rare Variants in Deoxyhypusine Synthase, an Enzyme Involved in the Synthesis of Hypusine, Are Associated with a Neurodevelopmental Disorder. Am. J. Hum. Genet. 104, 287-298.

Geloso, M.C., et al., 2017. The dual role of microglia in ALS: mechanisms and therapeutic approaches. Front. Aging Neurosci. 9, 242.

Gierga, K., et al., 2005. Involvement of the cranial nerves and their nuclei in spinocerebellar ataxia type 2 (SCA2). Acta Neuropathol. 109, 617-631.

Gispert, S., et al., 2012. The modulation of Amyotrophic Lateral Sclerosis risk by ataxin-2 intermediate polyglutamine expansions is a specific effect. Neurobiol. Dis. 45, 356-361.

Golomb, B.A., et al., 2009. Amyotrophic lateral sclerosis-like conditions in possible association with cholesterol-lowering drugs: an analysis of patient reports to the University of California, San Diego (UCSD) Statin Effects Study. Drug Saf. 32, 649-661.

Gonzalez De Aguilar, J.L., 2019. Lipid biomarkers for amyotrophic lateral sclerosis. Front. Neurol. 10, 284.

Goulding, D.R., et al., 2019. Inter-alpha-inhibitor deficiency in the mouse is associated with alterations in anxiety-like behavior, exploration and social approach. Genes Brain Behav. 18, e12505.

Grosskreutz, J., et al., 2010. Calcium dysregulation in amyotrophic lateral sclerosis. Cell Calcium 47, 165-174.

Guo, Z., et al., 2012. Tlx1/3 and Ptfla control the expression of distinct sets of transmitter and peptide receptor genes in the developing dorsal spinal cord. J. Neurosci. 32, 8509-8520.

Gutierrez, E., et al., 2013. eIF5A promotes translation of polyproline motifs. Mol. Cell 51, $35-45$.

Halbach, M.V., et al., 2017. Atxn2 Knockout and CAG42-Knock-in cerebellum shows similarly dysregulated expression in calcium homeostasis pathway. Cerebellum. 16, 68-81.

Hanrieder, J., Ewing, A.G., 2014. Spatial elucidation of spinal cord lipid- and metaboliteregulations in amyotrophic lateral sclerosis. Sci. Rep. 4, 5266.

Hart, M.P., Gitler, A.D., 2012. ALS-associated ataxin 2 polyQ expansions enhance stressinduced caspase 3 activation and increase TDP-43 pathological modifications. J. Neurosci. 32, 9133-9142.

Hartmann, G., 2017. Nucleic Acid Immunity. Adv. Immunol. 133, 121-169.

Haud, N., et al., 2011. rnaset2 mutant zebrafish model familial cystic leukoencephalopathy and reveal a role for RNase T2 in degrading ribosomal RNA. Proc. Natl. Acad. Sci. U. S. A. 108, 1099-1103.
Hawley, Z.C.E., et al., 2019. MiR-105 and miR-9 regulate the mRNA stability of neuronal intermediate filaments. Implications for the pathogenesis of amyotrophic lateral sclerosis (ALS). Brain Res. 1706, 93-100.

Hayashi, S., et al., 2013. Loss of calretinin- and parvalbumin-immunoreactive axons in anterolateral columns beyond the corticospinal tracts of amyotrophic lateral sclerosis spinal cords. J. Neurol. Sci. 331, 61-66.

Heck, M.V., et al., 2014. Dysregulated expression of lipid storage and membrane dynamics factors in Tia1 knockout mouse nervous tissue. Neurogenetics. 15, 135-144.

Helbig, K.L., et al., 2016. A recurrent mutation in KCNA2 as a novel cause of hereditary spastic paraplegia and ataxia. Ann. Neurol. 80, 638-642.

Heneka, M.T., et al., 2014. Innate immune activation in neurodegenerative disease. Nat. Rev. Immunol. 14, 463-477.

Henneke, M., et al., 2009. RNASET2-deficient cystic leukoencephalopathy resembles congenital cytomegalovirus brain infection. Nat. Genet. 41, 773-775.

Hou, L., et al., 2015. Intrathecal siRNA against GPNMB attenuates nociception in a rat model of neuropathic pain. J. Mol. Neurosci.: MN 55, 533-540.

Huang, J., et al., 2018. Regulation of mitochondrion-associated cytosolic ribosomes by mammalian mitochondrial ribonuclease T2 (RNASET2). J. Biol. Chem. 293, 19633-19644.

Huang, Y., et al., 2007. Neuronal growth and survival mediated by eIF5A, a polyaminemodified translation initiation factor. Proc. Natl. Acad. Sci. U. S. A. 104, 4194-4199.

Huttenrauch, M., et al., 2018. Glycoprotein NMB: a novel Alzheimer's disease associated marker expressed in a subset of activated microglia. Acta Neuropathol. Commun. 6, 108.

Ikeda, K., et al., 2013. Relationship between cervical cord 1H-magnetic resonance spectroscopy and clinoco-electromyographic profile in amyotrophic lateral sclerosis. Muscle Nerve 47, 61-67.

Ito, Y., et al., 2016. RIPK1 mediates axonal degeneration by promoting inflammation and necroptosis in ALS. Science. 353, 603-608.

Iwaki, H., et al., 2019. Genetic risk of Parkinson disease and progression: An analysis of 13 longitudinal cohorts. Neurol. Genet. 5, e348.

Jacobi, H., et al., 2015. Long-term disease progression in spinocerebellar ataxia types 1 , 2, 3, and 6: a longitudinal cohort study. Lancet Neurol. 14, 1101-1108.

Jagdeo, J.M., et al., 2018. N-terminomics TAILS identifies host cell substrates of poliovirus and Coxsackievirus B3 3C proteinases that modulate virus infection. J. Virol. 92, e02211-e02217.

Jiang, Y., et al., 2018. Upregulation of TREM2 ameliorates neuroinflammatory responses and improves cognitive deficits triggered by surgical trauma in Appswe/PS1dE9 Mice. Cell. Physiol. Biochem.: Intern. J. Exper. Cell. Physiol. Biochem. Pharmacol. 46, 1398-1411.

Kataoka, K., et al., 2013. The antinociceptive effect of SNAP5114, a gammaaminobutyric acid transporter-3 inhibitor, in rat experimental pain models. Anesth. Analg. 116, 1162-1169.

Key, J., et al., 2020. Mid-Gestation lethality of Atxn2l-Ablated Mice. Int. J. Mol. Sci. 21, 5124.

Kim, H.J., Taylor, J.P., 2017. Lost in transportation: nucleocytoplasmic transport defects in ALS and other neurodegenerative diseases. Neuron. 96, 285-297.

Kim, H.J., et al., 2008. Liver X receptor beta (LXRbeta): a link between beta-sitosterol and amyotrophic lateral sclerosis-Parkinson's dementia. Proc. Natl. Acad. Sci. U. S. A. $105,2094-2099$.

Klann, K., et al., 2020. Functional translatome proteomics reveal converging and dosedependent regulation by mTORC1 and eIF2alpha. Mol. Cell 77 (913-925), e4.

Koyano, S., et al., 2014. Neuropathological staging of spinocerebellar ataxia type 2 by semiquantitative $1 \mathrm{C} 2$-positive neuron typing. Nuclear translocation of cytoplasmic 1C2 underlies disease progression of spinocerebellar ataxia type 2. Brain Pathol. 24, 599-606.

Kozlov, G., et al., 2010. Structural basis of binding of P-body-associated proteins GW182 and ataxin- 2 by the Mlle domain of poly(A)-binding protein. J. Biol. Chem. 285, 13599-13606.

Kreilaus, F., et al., 2020. Novel behavioural characteristics of the superoxide dismutase 1 G93A (SOD1(G93A) mouse model of amyotrophic lateral sclerosis include sexdependent phenotypes. Genes Brain Behav. 19, e12604.

Kunde, S.A., et al., 2011. The X-chromosome-linked intellectual disability protein PQBP1 is a component of neuronal RNA granules and regulates the appearance of stress granules. Hum. Mol. Genet. 20, 4916-4931.

Kuo, P.H., et al., 2017. Dystonia and ataxia progression in spinocerebellar ataxias. Parkinsonism Relat. Disord. 45, 75-80.

Kuriakose, T., Kanneganti, T.D., 2018. ZBP1: innate sensor regulating cell death and inflammation. Trends Immunol. 39, 123-134.

Lahut, S., et al., 2012. ATXN2 and its neighbouring gene SH2B3 are associated with increased ALS risk in the Turkish population. PLoS One 7, e42956.

Lastres-Becker, I., et al., 2008. Insulin receptor and lipid metabolism pathology in ataxin2 knock-out mice. Hum. Mol. Genet. 17, 1465-1481.

Lastres-Becker, I., et al., 2016. Mammalian ataxin-2 modulates translation control at the pre-initiation complex via PI3K/mTOR and is induced by starvation. Biochim. Biophys. Acta 1862, 1558-1569.

Lastres-Becker, I., et al., 2019. New alternative splicing variants of the ATXN2 transcript. Neurol. Resear. Pract. 1, 22.

Lee, R.W., et al., 2013. Brain magnetic resonance imaging findings in Smith-Lemli-Opitz syndrome. Am. J. Med. Genet. A 161A, 2407-2419.

Lee, T., et al., 2011. Ataxin-2 intermediate-length polyglutamine expansions in European ALS patients. Hum. Mol. Genet. 20, 1697-1700.

Lehmann, S.M., et al., 2012. An unconventional role for miRNA: let-7 activates Toll-like receptor 7 and causes neurodegeneration. Nat. Neurosci. 15, 827-835. 
Li, C.H., et al., 2010. eIF5A promotes translation elongation, polysome disassembly and stress granule assembly. PLoS One 5, e9942.

Li, W., et al., 2015. N-acetyl-L-tryptophan delays disease onset and extends survival in an amyotrophic lateral sclerosis transgenic mouse model. Neurobiol. Dis. 80, 93-103.

Li, Y.R., et al., 2013. Stress granules as crucibles of ALS pathogenesis. J. Cell Biol. 201, 361-372.

Liapis, E., et al., 2012. Subcellular localization of regulator of G protein signaling RGS7 complex in neurons and transfected cells. J. Neurochem. 122, 568-581.

Lim, J., et al., 2006. A protein-protein interaction network for human inherited ataxias and disorders of Purkinje cell degeneration. Cell. 125, 801-814.

Lin, C.Y., et al., 2012. Reduced levels of interleukin 33 and increased levels of soluble ST2 in subjects with amyotrophic lateral sclerosis. J. Neuroimmunol. 249, 93-95.

Liu, J., et al., 2009. Deranged calcium signaling and neurodegeneration in spinocerebellar ataxia type 2. J. Neurosci. 29, 9148-9162.

Liu, Y., Szaro, B.G., 2011. hnRNP K post-transcriptionally co-regulates multiple cytoskeletal genes needed for axonogenesis. Development. 138, 3079-3090.

van de Loo, S., et al., 2009. Ataxin-2 associates with rough endoplasmic reticulum. Exp. Neurol. 215, 110-118.

Lu, B., et al., 2009. Peptide neurotransmitters activate a cation channel complex of NALCN and UNC-80. Nature. 457, 741-744.

Lubas, M., et al., 2018. eIF5A is required for autophagy by mediating ATG3 translation. EMBO Rep. 19, e46072.

Lui, H., et al., 2016. Progranulin deficiency promotes circuit-specific synaptic pruning by microglia via complement activation. Cell. 165, 921-935.

Mackay, D.S., et al., 2014. Methodological considerations for the harmonization of noncholesterol sterol bio-analysis. J. Chromatogr. B Anal. Technol. Biomed. Life Sci. 957, 116-122.

Maier, B., et al., 2010. The unique hypusine modification of eIF5A promotes islet beta cell inflammation and dysfunction in mice. J. Clin. Invest. 120, 2156-2170.

Mallaret, M., et al., 2014. The tumour suppressor gene WWOX is mutated in autosomal recessive cerebellar ataxia with epilepsy and mental retardation. Brain. 137, 411-419.

Mandal, A., et al., 2016. Global quantitative proteomics reveal up-regulation of endoplasmic reticulum stress response proteins upon depletion of eIF5A in HeLa cells. Sci. Rep. 6, 25795.

Maric, G., et al., 2015. GPNMB cooperates with neuropilin-1 to promote mammary tumor growth and engages integrin alpha5beta1 for efficient breast cancer metastasis. Oncogene. 34, 5494-5504.

Marin, M., et al., 2008. Human immunodeficiency virus type 1 Vif functionally interacts with diverse APOBEC3 cytidine deaminases and moves with them between cytoplasmic sites of mRNA metabolism. J. Virol. 82, 987-998.

Markovic, V., et al., 2016. Dystonia in patients with spinocerebellar Ataxia Type 2. Move. Disord. Clin. Pract. 3, 292-295.

Maskey, D., et al., 2010. Immunohistochemical localization of calbindin D28-k, parvalbumin, and calretinin in the cerebellar cortex of the circling mouse. Neurosci. Lett. 483, 132-136.

Mason, A.R., et al., 2017. The Receptor-interacting serine/Threonine protein kinase 1 (RIPK1) regulates progranulin levels. J. Biol. Chem. 292, 3262-3272.

Matsuishi, T., et al., 1999. Increased cerebrospinal fluid levels of substance P in patients with amyotrophic lateral sclerosis. Short Commun. J. Neural trans. 106, 943-948.

McCann, C., et al., 2011. The Ataxin-2 protein is required for microRNA function and synapse-specific long-term olfactory habituation. Proc. Natl. Acad. Sci. U. S. A. 108, E655-E662.

Medrano-Montero, J., et al., 2018. Early cranial nerve dysfunction is correlated to altered facial morphology in spinocerebellar ataxia type 2. Investig. Discapacid. 7, 53-66.

van Meer, G., de Kroon, A.I., 2011. Lipid map of the mammalian cell. J. Cell Sci. 124, 5-8.

Meierhofer, D., et al., 2016. Ataxin-2 (Atxn2)-knock-out mice show branched chain amino acids and fatty acids pathway alterations. Mol. Cell. Proteom.: MCP. 15, 1728-1739.

Miyazaki, K., et al., 2004. NEDL1, a novel ubiquitin-protein isopeptide ligase for dishevelled-1, targets mutant superoxide dismutase-1. J. Biol. Chem. 279, $11327-11335$.

Moloney, E.B., et al., 2018. The glycoprotein GPNMB is selectively elevated in the substantia nigra of Parkinson's disease patients and increases after lysosomal stress. Neurobiol. Dis. 120, 1-11.

Morganti, J.M., et al., 2016. Call off the $\operatorname{dog}(\mathrm{ma})$ : M1/M2 polarization is concurrent following traumatic brain injury. PLoS One 11, e0148001.

Mounce, B.C., et al., 2017. Polyamines and their role in virus infection. Microbiol, Mol. Biol. Rev: MMBR. 81 e00029-17.

Murugesan, V., et al., 2018. Validating glycoprotein non-metastatic melanoma B (gpNMB, osteoactivin), a new biomarker of Gaucher disease. Blood Cells Mol. Dis. $68,47-53$.

Nagahara, Y., et al., 2017. GPNMB ameliorates mutant TDP-43-induced motor neuron cell death. J. Neurosci. Res. 95, 1647-1665.

Netrakanti, P.R., et al., 2015. Fast cerebellar reflex circuitry requires synaptic vesicle priming by munc13-3. Cerebellum. 14, 264-283.

Newton, K., 2019. Multitasking Kinase RIPK1 Regulates Cell Death and Inflammation. Cold Spring Harb. Perspect. Biol. 12, a036368.

Nicolas, A., et al., 2018. Genome-wide analyses identify KIF5A as a novel ALS gene. Neuron. 97, 1268-1283 e6.

Nobrega, C., et al., 2019. Restoring brain cholesterol turnover improves autophagy and has therapeutic potential in mouse models of spinocerebellar ataxia. Acta Neuropathol. 138, 837-858.

Nonhoff, U., et al., 2007. Ataxin-2 interacts with the DEAD/H-box RNA helicase DDX6 and interferes with P-bodies and stress granules. Mol. Biol. Cell 18, 1385-1396.
Nonis, D., et al., 2008. Ataxin-2 associates with the endocytosis complex and affects EGF receptor trafficking. Cell. Signal. 20, 1725-1739.

Okroj, M., et al., 2012. Heavy chains of inter alpha inhibitor (IalphaI) inhibit the human complement system at early stages of the cascade. J. Biol. Chem. 287, 20100-20110.

Okuda, T., et al., 2003. PQBP-1 transgenic mice show a late-onset motor neuron diseaselike phenotype. Hum. Mol. Genet. 12, 711-725.

Oosthuyse, B., et al., 2001. Deletion of the hypoxia-response element in the vascular endothelial growth factor promoter causes motor neuron degeneration. Nat. Genet. $28,131-138$.

Orozco Diaz, G., et al., 1990. Autosomal dominant cerebellar ataxia: clinical analysis of 263 patients from a homogeneous population in Holguin, Cuba. Neurology 40, 1369-1375.

Ostrowski, L.A., et al., 2018. Conserved Pbp1/Ataxin-2 regulates retrotransposon activity and connects polyglutamine expansion-driven protein aggregation to lifespancontrolling rDNA repeats. Commun. Biol. 1, 187.

Oyama, F., et al., 2006. Sodium channel beta4 subunit: down-regulation and possible involvement in neuritic degeneration in Huntington's disease transgenic mice. J. Neurochem. 98, 518-529.

Paciorkowski, A.R., et al., 2011. Massive expansion of SCA2 with autonomic dysfunction, retinitis pigmentosa, and infantile spasms. Neurology. 77, 1055-1060.

Pandey, M., et al., 2017. A central role for R7bp in the regulation of itch sensation. Pain. 158, 931-944.

Park, H., et al., 2015. Parkinsonism in spinocerebellar ataxia. Biomed. Res. Int. 2015, 125273.

Parker, S.E., et al., 2019. Revisiting the role of the innate immune complement system in ALS. Neurobiol. Dis. 127, 223-232.

Paul, S., et al., 2018. Staufen1 links RNA stress granules and autophagy in a model of neurodegeneration. Nat. Commun. 9, 3648.

Paulson, H.L., et al., 2017. Polyglutamine spinocerebellar ataxias - from genes to potential treatments. Nat. Rev. Neurosci. 18, 613-626.

Pelosi, L., et al., 2019. Spinocerebellar ataxia type 2-neuronopathy or neuropathy? Muscle Nerve 60, 271-278.

Pena-Altamira, E., et al., 2016. Changing paradigm to target microglia in neurodegenerative diseases: from anti-inflammatory strategy to active immunomodulation. Expert Opin. Ther. Targets 20, 627-640.

Pereira, K.D., et al., 2016. Alternative start codon connects eIF5A to mitochondria. J. Cell. Physiol. 231, 2682-2689.

Perez, M.K., et al., 1998. Recruitment and the role of nuclear localization in polyglutamine-mediated aggregation. J. Cell Biol. 143, 1457-1470.

Ponomarev, I., et al., 2006. Transcriptional signatures of cellular plasticity in mice lacking the alpha1 subunit of GABAA receptors. J. Neurosci. 26, 5673-5683.

Porter, F.D., Herman, G.E., 2011. Malformation syndromes caused by disorders of cholesterol synthesis. J. Lipid Res. 52, 6-34.

Puleston, D.J., et al., 2019. Polyamines and eIF5A hypusination modulate mitochondrial respiration and macrophage activation. Cell Metab. 30, 352-363 e8.

Pulst, S.M., et al., 1996. Moderate expansion of a normally biallelic trinucleotide repeat in spinocerebellar ataxia type 2. Nat. Genet. 14, 269-276.

Rademakers, R., et al., 2012. Advances in understanding the molecular basis of frontotemporal dementia. Nat. Rev. Neurol. 8, 423-434.

Ralser, M., et al., 2005a. An integrative approach to gain insights into the cellular function of human ataxin-2. J. Mol. Biol. 346, 203-214.

Ralser, M., et al., 2005b. Ataxin-2 and huntingtin interact with endophilin-A complexes to function in plastin-associated pathways. Hum. Mol. Genet. 14, 2893-2909.

Rameshwar, P., 2012. The tachykinergic system as avenues for drug intervention. Recent Patents CNS Drug Discov. 7, 173-180.

Ransdell, J.L., et al., 2017. Loss of Navbeta4-mediated regulation of sodium currents in adult Purkinje neurons disrupts firing and impairs motor coordination and balance. Cell Rep. 19, 532-544.

Ransohoff, R.M., 2016. A polarizing question: do M1 and M2 microglia exist? Nat. Neurosci. 19, 987-991.

Reetz, K., et al., 2018. Brain atrophy measures in preclinical and manifest spinocerebellar ataxia type 2. Ann. Clin. Transl. Neurol. 5, 128-137.

Riess, O., et al., 1997. SCA2 trinucleotide expansion in German SCA patients. Neurogenetics. 1, 59-64.

Rodriguez-Labrada, R., et al., 2016. Spinocerebellar ataxia type 2: measures of saccade changes improve power for clinical trials. Move. Disord: Off. J. Move. Disord. Soc. $31,570-578$.

Rodriguez-Labrada, R., et al., 2019. Sleep spindles and K-complex activities are decreased in spinocerebellar ataxia type 2: relationship to memory and moto performances. Sleep Med. 60, 188-196.

Ross, O.A., et al., 2011. Ataxin-2 repeat-length variation and neurodegeneration. Hum. Mol. Genet. 20, 3207-3212.

Rossi, D., et al., 2014. eIF5A has a function in the cotranslational translocation of proteins into the ER. Amino Acids 46, 645-653.

Rub, U., et al., 2003a. Thalamic involvement in a spinocerebellar ataxia type 2 (SCA2) and a spinocerebellar ataxia type 3 (SCA3) patient, and its clinical relevance. Brain J. Neurol. 126, 2257-2272.

Rub, U., et al., 2003b. Anatomically based guidelines for systematic investigation of the central somatosensory system and their application to a spinocerebellar ataxia type 2 (SCA2) patient. Neuropathol. Appl. Neurobiol. 29, 418-433.

Rub, U., et al., 2006. Degeneration of ingestion-related brainstem nuclei in spinocerebellar ataxia type 2, 3,6 and 7. Neuropathol. Appl. Neurobiol. 32, 635-649.

Rub, U., et al., 2007. Consistent affection of the central somatosensory system in spinocerebellar ataxia type 2 and type 3 and its significance for clinical symptoms and rehabilitative therapy. Brain Res. Rev. 53, 235-249. 
Rub, U., et al., 2013. Clinical features, neurogenetics and neuropathology of the polyglutamine spinocerebellar ataxias type 1, 2, 3, 6 and 7. Prog. Neurobiol. 104, 38-66.

Rubino, E., et al., 2019. ATXN2 intermediate repeat expansions influence the clinical phenotype in frontotemporal dementia. Neurobiol. Aging 73, 231 e7-231 e9.

Sangaraju, D., et al., 2017. A multi-matrix HILIC-MS/MS method for the quantitation of endogenous small molecule neurological biomarker $\mathrm{N}$-acetyl aspartic acid (NAA). J. Pharm. Biomed. Anal. 140, 11-19.

Satoh, J., Yamamura, T., 2004. Gene expression profile following stable expression of the cellular prion protein. Cell. Mol. Neurobiol. 24, 793-814.

Satoh, J.I., et al., 2019. Microglia express GPNMB in the brains of Alzheimer's disease and Nasu-Hakola disease. Intract. Rare Dis. Resear. 8, 120-128.

Satterfield, T.F., Pallanck, L.J., 2006. Ataxin-2 and its Drosophila homolog, ATX2, physically assemble with polyribosomes. Hum. Mol. Genet. 15, 2523-2532.

Satterfield, T.F., et al., 2002. A Drosophila homolog of the polyglutamine disease gene SCA2 is a dosage-sensitive regulator of actin filament formation. Genetics. 162, 1687-1702.

Schaaf, C.P., et al., 2011. Desmosterolosis-phenotypic and molecular characterization of a third case and review of the literature. Am. J. Med. Genet. A 155A, 1597-1604.

Scherzed, W., et al., 2012. Pathoanatomy of cerebellar degeneration in spinocerebellar ataxia type 2 (SCA2) and type 3 (SCA3). Cerebellum. 11, 749-760.

Schindelin, J., et al., 2012. Fiji: an open-source platform for biological-image analysis. Nat. Methods 9, 676-682.

Schmittgen, T.D., Livak, K.J., 2008. Analyzing real-time PCR data by the comparative C (T) method. Nat. Protoc. 3, 1101-1108.

Schols, L., et al., 1997. Spinocerebellar ataxia type 2. Genotype and phenotype in German kindreds. Arch. Neurol. 54, 1073-1080.

Schols, L., et al., 2015. No parkinsonism in SCA2 and SCA3 despite severe neurodegeneration of the dopaminergic substantia nigra. Brain J. Neurol. 138, 3316-3326.

Scoles, D.R., Pulst, S.M., 2019. Antisense therapies for movement disorders. Mov. Disord. 34, 1112-1119.

Scoles, D.R., et al., 2017. Antisense oligonucleotide therapy for spinocerebellar ataxia type 2. Nature. 544, 362-366.

Scoles, D.R., et al., 2020. ALS-associated genes in SCA2 mouse spinal cord transcriptomes. Hum. Mol. Genet. 29, 1658-1672.

Seidel, G., et al., 2017a. Quantitative global proteomics of yeast PBP1 deletion mutants and their stress responses identifies glucose metabolism, mitochondrial, and stress granule changes. J. Proteome Res. 16, 504-515.

Seidel, K., et al., 2017b. On the distribution of intranuclear and cytoplasmic aggregates in the brainstem of patients with spinocerebellar ataxia type 2 and 3. Brain Pathol. 27, 345-355.

Sen, N.E., et al., 2016. Search for SCA2 blood RNA biomarkers highlights Ataxin-2 as strong modifier of the mitochondrial factor PINK1 levels. Neurobiol. Dis. 96, $115-126$.

Sen, N.E., et al., 2017. PINK1 and Ataxin-2 as modifiers of growth. Oncotarget. 8, 32382-32383.

Sen, N.E., et al., 2019a. In human and mouse spino-cerebellar tissue, Ataxin-2 expansion affects ceramide-sphingomyelin metabolism. Int. J. Mol. Sci. 20, 5854.

Sen, N.E., et al., 2019b. Generation of an Atxn2-CAG100 knock-in mouse reveals Nacetylaspartate production deficit due to early Nat81 dysregulation. Neurobiol. Dis. $132,104559$.

Shapouri, F., et al., 2016. Tob1 is expressed in developing and adult gonads and is associated with the P-body marker, Dcp2. Cell Tissue Res. 364, 443-451.

Shulman, J.M., Feany, M.B., 2003. Genetic modifiers of tauopathy in Drosophila. Genetics. 165, 1233-1242.

Singh, H.N., Rajeswari, M.R., 2015. Role of long purine stretches in controlling the expression of genes associated with neurological disorders. Gene. 572, 175-183.

Singleton, A.B., et al., 2003. alpha-Synuclein locus triplication causes Parkinson's disease. Science. 302, 841.

Sirianni, A.C., et al., 2015. N-acetyl-1-tryptophan, but not N-acetyl-d-tryptophan, rescues neuronal cell death in models of amyotrophic lateral sclerosis. J. Neurochem. 134, 956-968.

Sliter, D.A., et al., 2018. Parkin and PINK1 mitigate STING-induced inflammation. Nature. 561, 258-262.

Sosic-Jurjevic, B., et al., 2019. The isoflavones genistein and daidzein increase hepatic concentration of thyroid hormones and affect cholesterol metabolism in middle-aged male rats. J. Steroid Biochem. Mol. Biol. 190, 1-10.

Stallings, N.R., et al., 2013. TDP-43, an ALS linked protein, regulates fat deposition and glucose homeostasis. PLoS One 8, e71793.

Stamelou, M., et al., 2014. The phenotypic spectrum of DYT24 due to ANO3 mutations. Move. Disord: Off. J. Move. Disord. Soc. 29, 928-934.

Stribl, C., et al., 2014. Mitochondrial dysfunction and decrease in body weight of a transgenic knock-in mouse model for TDP-43. J. Biol. Chem. 289, 10769-10784.

Sudhakaran, I.P., et al., 2014. FMRP and Ataxin-2 function together in long-term olfactory habituation and neuronal translational control. Proc. Natl. Acad. Sci. U. S A. 111, E99-E108.

Tanaka, H., et al., 2012. The potential of GPNMB as novel neuroprotective factor in amyotrophic lateral sclerosis. Sci. Rep. 2, 573.
Taylor, J.P., et al., 2016. Decoding ALS: from genes to mechanism. Nature. 539, 197-206.

Taylor, L.M., et al., 2018. Pathological phosphorylation of tau and TDP-43 by TTBK1 and TTBK2 drives neurodegeneration. Mol. Neurodegener. 13, 7.

Thelen, K.M., et al., 2006. Brain cholesterol synthesis in mice is affected by high dose of simvastatin but not of pravastatin. J. Pharmacol. Exp. Ther. 316, 1146-1152.

Thiagarajan, D., et al., 2011. The DNA methyltranferase Dnmt2 participates in RNA processing during cellular stress. Epigenetics. 6, 103-113.

Tollervey, J.R., et al., 2011. Characterizing the RNA targets and position-dependent splicing regulation by TDP-43. Nat. Neurosci. 14, 452-458.

Toonen, L.J.A., et al., 2018. Transcriptional profiling and biomarker identification reveal tissue specific effects of expanded ataxin-3 in a spinocerebellar ataxia type 3 mouse model. Mol. Neurodegener. 13, 31.

Torres-Odio, S., et al., 2017. Progression of pathology in PINK1-deficient mouse brain from splicing via ubiquitination, ER stress, and mitophagy changes to neuroinflammation. J. Neuroinflammation 14, 154.

Toyoshima, Y., et al., 2011. Spinocerebellar ataxia type 2 (SCA2) is associated with TDP43 pathology. Acta Neuropathol. 122, 375-378.

Uchihara, T., et al., 2001. Non-expanded polyglutamine proteins in intranuclear inclusions of hereditary ataxias-triple-labeling immunofluorescence study. Acta Neuropathol. 102, 149-152.

Varjosalo, M., et al., 2013. Interlaboratory reproducibility of large-scale human proteincomplex analysis by standardized AP-MS. Nat. Methods 10, 307-314.

Vejux, A., et al., 2018. Biomarkers of amyotrophic lateral sclerosis: current status and interest of oxysterols and phytosterols. Front. Mol. Neurosci. 11, 12.

Velazquez-Perez, L., et al., 2001. Hereditary ataxias in Cuba. Historical, epidemiological, clinical, electrophysiological and quantitative neurological features. Rev. Neurol. 32, 71-76.

Velazquez-Perez, L., et al., 2004. Saccade velocity is controlled by polyglutamine size in spinocerebellar ataxia 2. Ann. Neurol. 56, 444-447.

Velazquez-Perez, L., et al., 2009. Saccade velocity is reduced in presymptomatic spinocerebellar ataxia type 2. Clin. Neurophysiol.: Off. J. Intern. Feder. Clin. Neurophysiol. 120, 632-635.

Velazquez-Perez, L., et al., 2010. Progression markers of Spinocerebellar ataxia 2. A twenty years neurophysiological follow up study. J. Neurol. Sci. 290, 22-26.

Velazquez-Perez, L., et al., 2011. Sleep disorders in spinocerebellar ataxia type 2 patients. Neurodegener. Dis. 8, 447-454.

Velazquez-Perez, L., et al., 2014. Progression of early features of spinocerebellar ataxia type 2 in individuals at risk: a longitudinal study. Lancet Neurol. 13, 482-489.

Velazquez-Perez, L., et al., 2016a. Abnormal corticospinal tract function and motor cortex excitability in non-ataxic SCA2 mutation carriers: A TMS study. Clin. Neurophysiol.: Off. J. Intern. Feder. Clin. Neurophysiol. 127, 2713-2719.

Velazquez-Perez, L., et al., 2016b. Central motor conduction time as prodromal biomarker in spinocerebellar ataxia type 2. Move. Disord: Off. J. Move. Disord. Soc. 31, 603-604.

Velazquez-Perez, L., et al., 2017a. Corticomuscular coherence: a novel tool to assess the pyramidal tract dysfunction in spinocerebellar ataxia type 2. Cerebellum. 16, 602-606.

Velazquez-Perez, L., et al., 2017b. Early corticospinal tract damage in prodromal SCA2 revealed by EEG-EMG and EMG-EMG coherence. Clin. Neurophysiol.: Off. J. Intern. Feder. Clin. Neurophysiol. 128, 2493-2502.

Velazquez-Perez, L., et al., 2018. Progression of corticospinal tract dysfunction in preataxic spinocerebellar ataxia type 2: a two-years follow-up TMS study. Clin. Neurophysiol.: Off. J. Intern. Feder. Clin. Neurophysiol. 129, 895-900.

Wadia, N.H., Swami, R.K., 1971. A new form of heredo-familial spinocerebellar degeneration with slow eye movements (nine families). Brain J. Neurol. 94, 359-374.

Wang, L., et al., 2015. Large-scale assessment of polyglutamine repeat expansions in Parkinson disease. Neurology. 85, 1283-1292.

Waragai, M., et al., 1999. PQBP-1, a novel polyglutamine tract-binding protein, inhibits transcription activation by Brn-2 and affects cell survival. Hum. Mol. Genet. 8, 977-987.

Wechsler, A., et al., 2003. Generation of viable cholesterol-free mice. Science. 302, 2087.

West, A.P., Shadel, G.S., 2017. Mitochondrial DNA in innate immune responses and inflammatory pathology. Nat. Rev. Immunol. 17, 363-375.

White, J.P., Lloyd, R.E., 2011. Poliovirus unlinks TIA1 aggregation and mRNA stress granule formation. J. Virol. 85, 12442-12454.

Wishart, T.M., et al., 2012. Combining comparative proteomics and molecular genetics uncovers regulators of synaptic and axonal stability and degeneration in vivo. PLoS Genet. 8, e1002936.

Xie, G., et al., 2010. A new Kv1.2 channelopathy underlying cerebellar ataxia. J. Biol. Chem. 285, 32160-32173.

Xu, D., et al., 2018. TBK1 suppresses RIPK1-driven apoptosis and inflammation during development and in aging. Cell. 174, 1477-1491 e19.

Yoh, S.M., et al., 2015. PQBP1 Is a proximal sensor of the cGAS-dependent innate response to HIV-1. Cell. 161, 1293-1305.

Yokoshi, M., et al., 2014. Direct binding of Ataxin-2 to distinct elements in 3' UTRs promotes mRNA stability and protein expression. Mol. Cell 55, 186-198. 
Yousef, G.M., et al., 1999. Molecular characterization of zyme/protease M/neurosin (PRSS9), a hormonally regulated kallikrein-like serine protease. Genomics. 62 251-259.

Yuan, J., et al., 2019. Necroptosis and RIPK1-mediated neuroinflammation in CNS diseases. Nat. Rev. Neurosci. 20, 19-33.

Zhang, H., et al., 2019. Polyamines control eIF5A hypusination, TFEB translation, and autophagy to reverse B cell senescence. Mol. Cell 76, 110-125 e9.

Zhang, K., et al., 2018. Stress granule assembly disrupts nucleocytoplasmic transport. Cell. 173 (958-971), e17.

Zhang, L., et al., 2011. Muscle atrophy and motor neuron degeneration in human NEDL1 transgenic mice. J. Biomed. Biotechnol. 2011, 831092
Zhang, X.Y., et al., 2017. Mutations of PQBP1 in Renpenning syndrome promote ubiquitin-mediated degradation of FMRP and cause synaptic dysfunction. Hum. Mol Genet. 26, 955-968.

Zhang, Y.J., et al., 2007. Progranulin mediates caspase-dependent cleavage of TAR DNA binding protein-43. J. Neurosci. 27, 10530-10534.

Zhao, W., et al., 2015. TDP-43 activates microglia through NF-kappaB and NLRP3 inflammasome. Exp. Neurol. 273, 24-35.

Zieglgansberger, W., 2019. Substance P and pain chronicity. Cell Tissue Res. 375, 227-241. 